\title{
TWO NEW SPECIES OF THE SUPERFAMILY PHTHIRACAROIDEA (ACARI, ORIBATIDA) FROM THE SEYCHELLES AND THE USA WITH NOTES ON OTHER PTYCTIMOUS MITES FROM DIVERSE COUNTRIES
}

\author{
Wojciech NiedbaeA ${ }^{1}$ and Josef Starý ${ }^{2}$ \\ ${ }^{1}$ Department of Animal Taxonomy and Ecology, Faculty of Biology, Adam Mickiewicz University \\ in Poznań, Umultowska 89, 61-614 Poznań, Poland; E-mail: wojciech.niedbala@amu.edu.pl \\ ${ }^{2}$ Biology Centre, Academy of Sciences of the Czech Republic v.v.i., Institute of Soil Biology \\ Na Sádkách 7, CZ-37005 České Budějovice, Czech Republic; E-mail: jstary@upb.cas.cz
}

Two new species of the family Phthiracaridae, Arphthicarus paratinctus sp. $\mathrm{n}$. from the Seychelles, and Atropacarus (Atropacarus) paraclavigerus sp. n. from California, USA are described and figured. A comparison of morphological similarities with the most closely related species is presented. Additional descriptions, morphological remarks and taxonomical notes for seven ptyctimous species: Arphthicarus sculptilis (Niedbała, 1988), Austrophthiracarus diazae (Ojeda, 1985), Mesoplophora (Mesoplophora) parabacilla Niedbała, 2006, Notophthiracarus lienhardi Mahunka, 1996, N. stenotus Niedbała et Corpuz-Raros, 1998, Plonaphacarus semiaciculatus (Mahunka, 2008), and Steganacarus (Rhacaplacarus) brasiliensis (Pérez-Iñigo et Baggio, 1980) were added. In total, 105 species of ptyctimous oribatid mites were determined in material of 1910 specimens from 15 countries - Bangladesh, Brazil, Canada, Colombia, Cuba, Galapagos, Gough Island, Indonesia (Irian), Jamaica, Kenya, Malaysia, Reunion, Seychelles, Tanzania and the USA. Fauna of ptyctimous mites of Bangladesh, Colombia and Gough Island was studied for the first time. New records of ptyctimous mites for Brazil, Canada, Cuba, Jamaica, Malaysia, New Guinea (Indonesia, Irian), Reunion, Tanzania and the USA are also presented. A replacement name for the junior homonym Austrophthiracarus longisetosus Niedbała et Starý, 2015, A. longisetus nom. n. is proposed.

Key words: ptyctimous mites, new species, taxonomy, new records, distribution, oribatid mites.

\section{INTRODUCTION}

Many interesting findings from different zoogeographical regions were recorded during the study of a large number of ptyctimous mites deposited at the Institute of Soil Biology, BC ASCR, České Budějovice, Czech Republic. Two new species were identified in the material collected in leaf litter in mixed forest from Mahé Island, Seychelles and in the spruce litter in Patrick's Point State Park, California, USA. The description of two new species of ptyctimous mites is the subject of this contribution. Both these regions (Californian Floristic Province and Seychelles together with Madagascar) are included among the most important biodiversity hotspots in the world characterized by a high 
concentration of endemic taxa (Myers et al. 2000). Our knowledge on the fauna of ptyctimous mites of the Seychelles is incomplete. NiedbaŁa (2010) summarized the ptyctimous fauna of the Seychelles and recorded from there nine species in total; one species from the family Mesoplophoridae, two species from the family Oribotritiidae, one species from the family Euphthiracaridae, two species from the family Phthiracaridae and three species from the family Steganacaridae. More species of ptyctimous mites were recorded from California, USA. Niedba€A (2002) surveyed the fauna of ptyctimous mites from the Nearctic Region and recorded one species from the family Protoplophoridae, two species from the family Mesoplophoridae, and 11 species from the superfamily Phthiracaroidea in California. Also 16 species of the superfamily Euphthiracaroidea were recorded from Californian soils and nine species from them were described by WALKER (1965).

\section{MATERIAL AND METHODS}

Leaf litter and soil samples were extracted by using modified Tullgren funnels. All the extracted mite specimens were preserved in $85 \%$ ethanol, then cleared in $80 \%$ lactic acid on temporary cavity slides and mounted on temporary slides with glycerol. The determined material was preserved in vials with $80 \%$ ethanol. Observations, figures, and measurements were made using a standard light microscope equipped with a drawing attachment. All the measurements are given in micrometres. The terminology used is based on that of Niedbaєa (2000). Type material is partly deposited at the Department of Animal Taxonomy and Ecology, Poznań, Poland (DATE) and partly at the Institute of Soil Biology BC ASCR, České Budějovice, in the Czech Republic (ISB), and partly at the Natural History Museum, Geneva, Switzerland (NHMG).

\section{DESCRIPTION OF NEW SPECIES}

\section{Arphthicarus paratinctus sp. n.}

(Figs 1A-E)

Material examined - Holotype deposited at DATE from the locus typicus: SEY-005, Seychelles, Mahé, Morne, 3.XII.1975, 750 m, deciduous forest, leaf litter sample, leg A. Fjellberg.

Etymology - The name of the species, paratinctus, alludes to the similarity of the new species to Arphthicarus tinctus Niedbała, 2000.

Measurements of holotype - Prodorsum: length 202, width 134, height 73, sensillus 20, length of prodorsal setae: interlamellar (in) 28, lamellar (le) 20, rostral (ro) 35; notogaster: length 343 , width 227 , height 217 , length of notogastral setae: $c_{1} 61, h_{1} 51, p s_{1} 48$; genitoaggenital plate $88 \times 73$, anoadanal plate $126 \times 76$. 
Description - Colour light brown. Cuticle well sculptured with weak concavities.

Prodorsum with sigillar fields not very distinct, median field large. Lateral carinae absent. Sensilli with short, narrow pedicel and globular head, rough. Prodorsal setae very short, spiniform, rough, rostral setae (ro) the longest.

Notogaster with 15 pairs of short spiniform, rough notogastral setae tapering distally, $c_{1} / c_{1}-d_{1}=0.75$. Setae $c_{1}$ near anterior border, setae $c_{3}$ slightly remote and setae $c_{2}$ far from anterior notogastral border. Vestigial setae invisible. Three pairs of lyrifissures im, ip and ips present.

Ventral region. Setae $h$ of mentum slightly longer than distance between them.

Genitoaggenital plates with nine pairs of genital setae with arrangement: 4+2: 3 . Anoadanal plates each with five pairs of setae, setae $a d_{3}$ smallest, setae $a d_{2}$ longest and situated near setae $a d_{1}$ and near paraxial border of plate.

Legs. Formulae of setae and solenidia of complete type. Setae $d$ of femora I long and considerably remote from distal end of article, setae $l$ " situated near distal end.

Comparison - The new species is slightly similar to Arphthicarus tinctus Niedbała, 2000 from New Caledonia in the shape of the sensilli, length of prodorsal setae, implantation and arrangement of setae of femora I, but is distinguishable by the shape of the notogastral setae and the arrangement of genital, anal and adanal setae (NiedBAEA 2000).

\section{Atropacarus (Atropacarus) paraclavigerus sp. n.}

(Figs 2A-I)

Material examined - Holotype deposited at DATE from the locus typicus: USA-195, California, Humboldt County, Patrick's Point State Park, 21.VI.1991, 41ํㅇ' N, $124^{\circ} 10^{\prime} \mathrm{W}$, coniferous forest with spruce Picea sitchensis, sample of coniferous litter and soil, leg K. Horner.

Etymology - The specific epithet, paraclavigerus, alludes to the resemblance of the new species to Atropacarus (Atropacarus) clavigerus (Berlese, 1904).

Measurements of holotype - Prodorsum: length 202, width 126, height 86, sensillus 68, length of prodorsal setae: interlamellar (in) 23, lamellar (le) 15, rostral (ro) 20, exobothridial (ex) 10; notogaster: length 348 , width 227 , height 207, length of notogastral setae: $c_{1} 18, h_{1} 23, p s_{1} 18$; genitoaggenital plate $81 \times 58$, anoadanal plate $81 \times 40$.

Description - Colour light grey. Cuticle well sculptured with distinct concavities.

Prodorsum with long and narrow sigillar fields. Lateral carinae distinct. Sensilli sickle-shaped, covered with small spines at distal half. Prodorsal setae short, covered with minute and dense spines, their relative lengths in $>$ ro $>l e>e x$.

Notogaster with 21 pairs of notogastral setae, additional setae in rows $c$, $h$ and $p s$, very short $\left(c_{1}<1 / 4 c_{1}-d_{1}\right)$, densely covered with spines, tuft-like. Setae $c_{1-3}$ far from the anterior notogastral margin, with $c_{2}$ furthest away. Vestigial setae invisible because of strong sculpture. Two pairs of lyrifissures ( $\mathrm{i} a$ and $\mathrm{im}$ ) present.

Ventral region. Setae $h$ of mentum shorter than distance between them. Anoadanal plates with minute setae, $a n /$ not $=0.02$. Setation of legs complete; $v^{\prime \prime} / v^{\prime}=4$. 


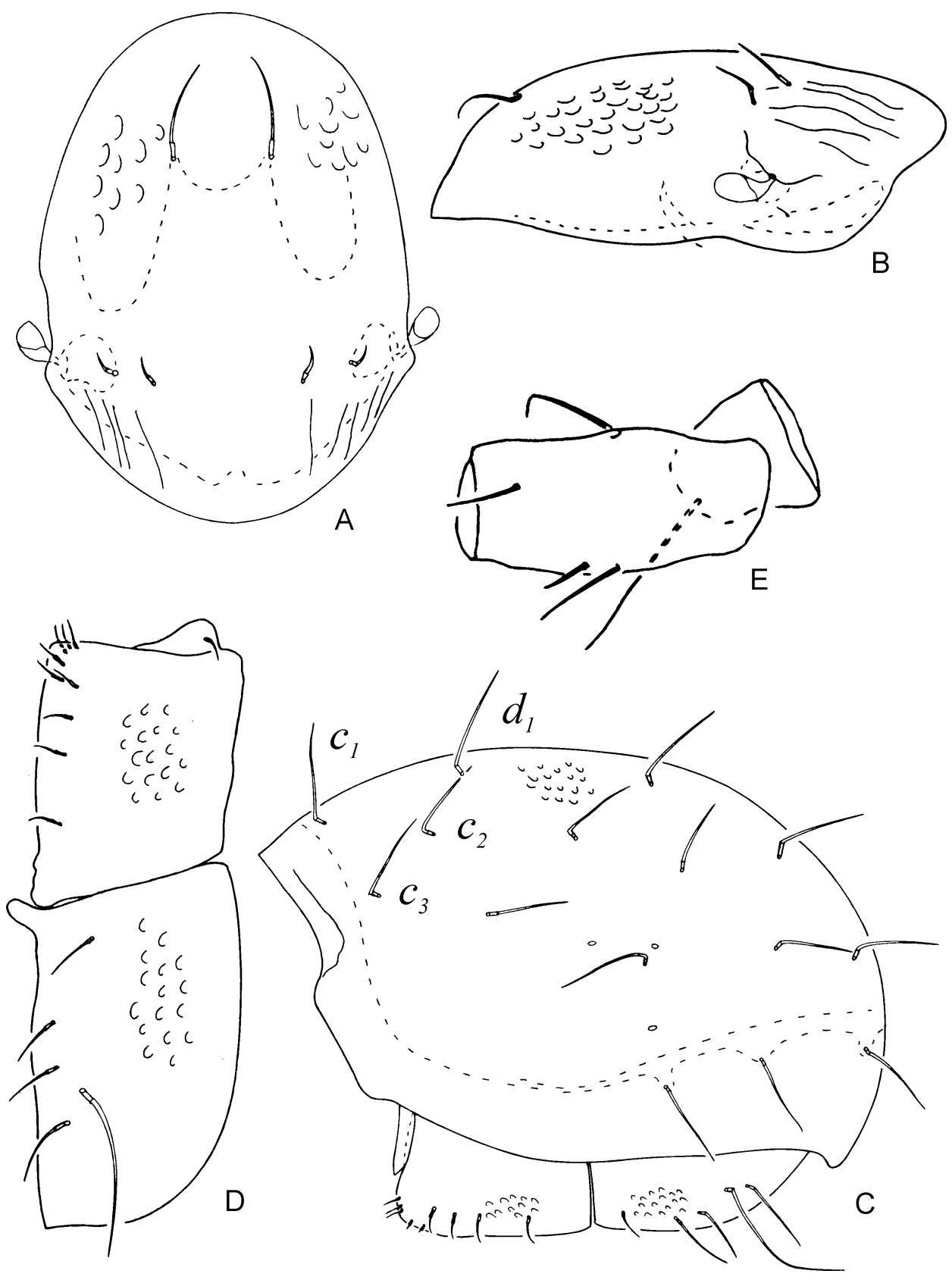

Figs 1A-E. Arphthicarus paratinctus sp. n.: $\mathrm{A}=$ prodorsum, dorsal view, $\mathrm{B}=$ prodorsum, lateral view, $\mathrm{C}=$ opisthosoma, lateral view, $\mathrm{D}=$ genitoaggenital and anoadanal plates, $\mathrm{E}=$ trochanter and femur of leg I. 


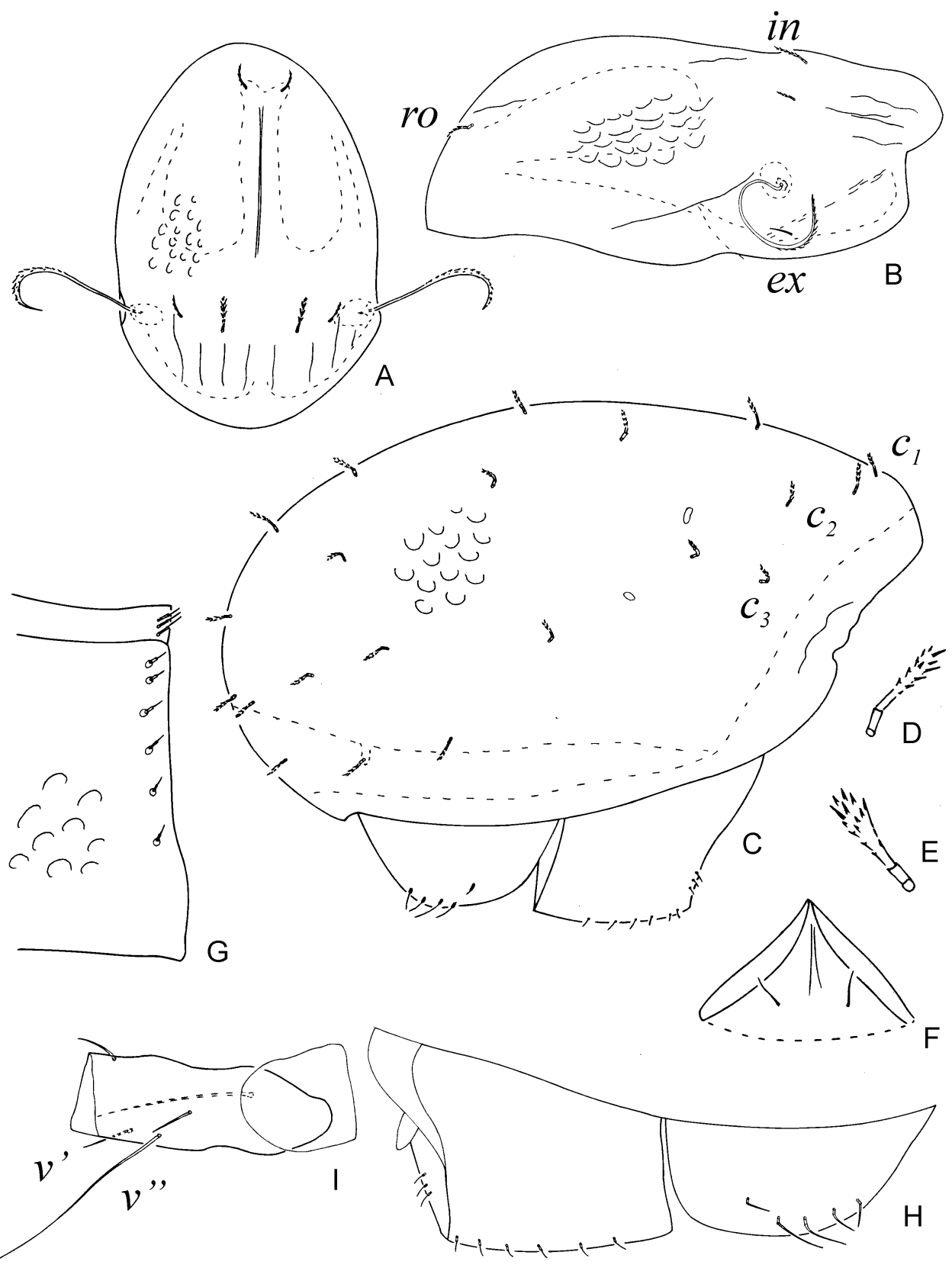

Figs 2A-I. Atropacarus (Atropacarus) paraclavigerus sp. n.: A = prodorsum, dorsal view, B = prodorsum, lateral view, $\mathrm{C}=$ opisthosoma, lateral view, $\mathrm{D}=$ seta $c_{1}, \mathrm{E}=$ seta $h_{1}, \mathrm{~F}=$ mentum of subcapitulum, $\mathrm{G}=$ fragment of genitoaggenital plate, $\mathrm{H}=$ genitoaggenital and anoadanal plates, lateral view, $\mathrm{I}=$ trochanter and femur of leg $\mathrm{I}$. 
Comparison - The new species is very similar to the Western Palaearctic species Atropacarus (Atropacarus) clavigerus (Berlese, 1904). The morphological differences are very small: the number (21) of notogastral setae (in $A$. $(A$.) clavigerus 20 pairs), setae $h$ of mentum shorter than the distance between them (longer in $A$. (A.) clavigerus) and setae $l$ " of femora I inserted more posteriorly than in A. (A.) clavigerus (see Niedba€A 2011).

\title{
ADDITIONAL DESCRIPTIONS AND TAXONOMICAL NOTES WITH SOME MORPHOLOGICAL REMARKS
}

\author{
Mesoplophora (Mesoplophora) parabacilla Niedbała, 2006 \\ (Figs 3A-D)
}

Material examined - Two specimens deposited at DATE from the locality: COL-009, Colombia, Meta, Carimagua, 15.IV.1983, native pasture, savannas, hand sampling, leg J. M. Guerrero.

Measurements of one specimen - Length of prodorsum 364, length of notogaster 520. Exobothridial setae (ex) longer than diameter of bothridia.

Remarks - New for the fauna of Colombia. Specimens from Colombia are slightly larger than specimens from Panama (NiedbaŁa 2006).

\section{Plonaphacarus semiaciculatus (Mahunka, 2008)}

(Figs $4 \mathrm{~A}-\mathrm{G}$ )

Rhacaplacarus (Rhacaplacarus) semiaciculatus Mahunka, 2008

Material examined - One specimen deposited at DATE: MAY-044, West Malaysia, Batu Caves, 30.XII.2010, $03^{\circ} 14^{\prime} 5^{\prime \prime} \mathrm{N}, 101^{\circ} 41^{\prime} 17^{\prime \prime} \mathrm{E}$, rain forest on lime-stone bedrock, wet site with rich brushwood, sample of leaf litter, leg J. Farská.

Diagnosis - Surface of body ornamented by large alveoli. Prodorsum with median crista and long, lateral carinae; sensilli long, with club-like head; lamellar setae very short, rough; rostral setae straight, rough; exobothridial setae vestigial; all adanal setae longer than anal setae, setae $a d_{2}$ bent distally.

Measurements of specimen from sample MAY-044 - Prodorsum: length 263, width 197, height 126, sensillus 66, length of prodorsal setae: interlamellar (in) 139, lamellar (le) 28 , rostral (ro) 121; notogaster: length 525, width 374, height 348 , length of notogastral setae: $c_{1} 164, c_{1} / c_{1}-d_{1}=1.1, h_{1}$ and $p s_{1} 185$; genitoaggenital plate $164 \times 96$; anoadanal plate $164 \times 101$.

Redescription - Colour dark brown. Integument with deep and large alveoli.

Prodorsum with weak sigillar fields, median field with anterior incision. Median crista present. Lateral carinae long, reaching anterior part of prodorsum. Sensilli long, with 
club-like head. Interlamellar setae (in) long, erect, spinose in distal half, lamellar setae (le) short, rough, rostral setae (ro) straight, rough, exobothridial (ex) setae vestigial.

Notogaster with 15 pairs of rigid notogastral setae, relatively long, their mutual length $c_{1}>c_{1}-d_{1}$, covered with small spines in distal half; dorsal setae slightly longer than lateral; setae $c_{1}$ and $c_{3}$ near anterior margin, setae $c_{1}$ more than setae $c_{3}$, setae $c_{2}$ far from margin. Vestigial setae and lyrifissures not observed because of strong sculpture.
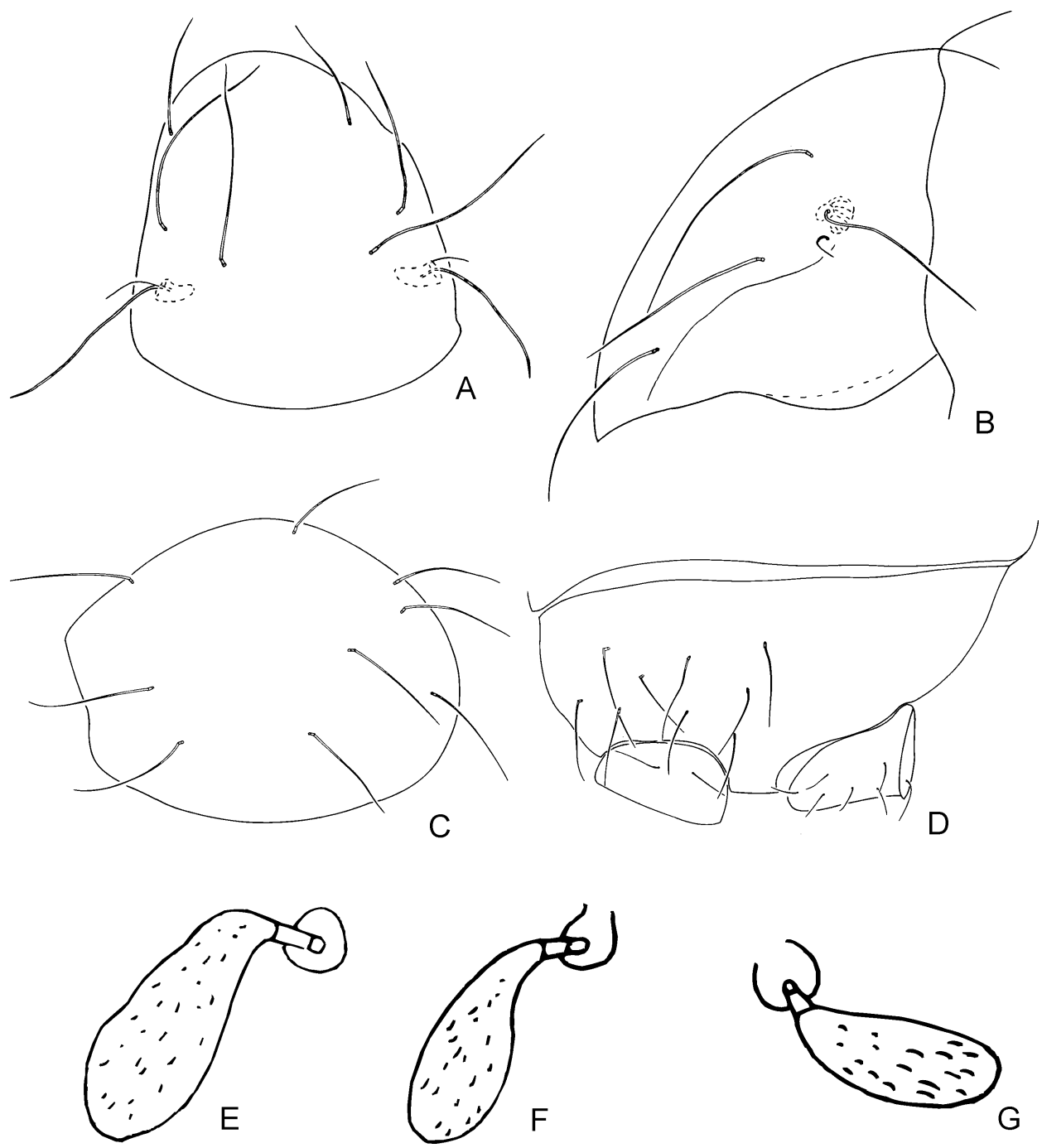

Figs 3A-G. A-D. Mesoplophora (Mesoplophora) parabacilla Niedbała, 2006, specimen from COL-009: $\mathrm{A}=$ prodorsum, dorsal view, $\mathrm{B}=$ prodorsum, lateral view, $\mathrm{C}=$ notogaster, laterodorsal view, D = ventral plate, lateral view. E-G. Notophthiracarus stenotus Niedbała et

Corpuz-Raros, 1998, specimen from NG-1803: $\mathrm{E}=\operatorname{seta} c_{2^{\prime}} \mathrm{F}=$ seta $d_{2^{\prime}} \mathrm{G}=$ seta $p s_{1}$. 

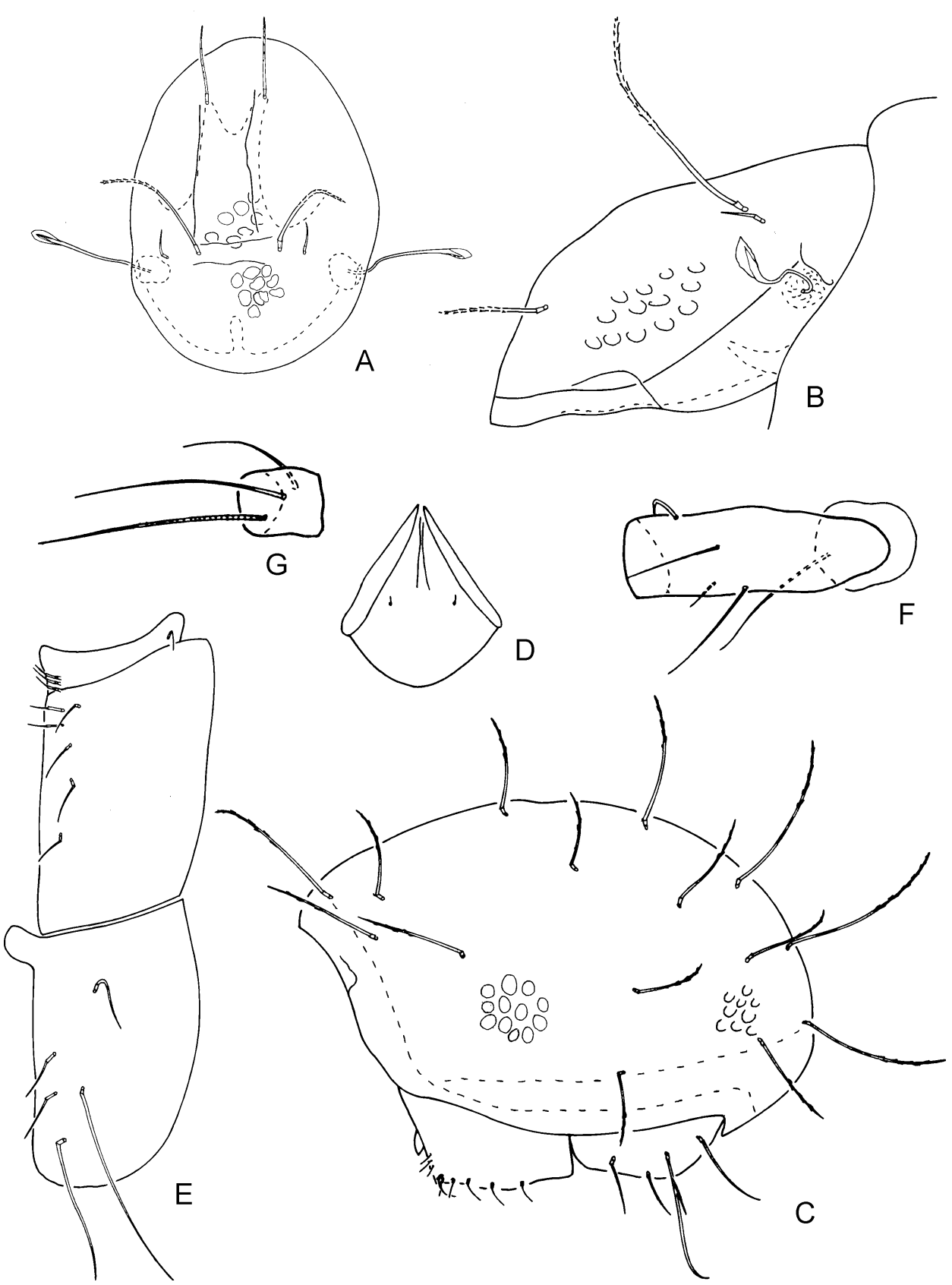

Figs 4A-G. Plonaphacarus semiaciculatus (Mahunka, 2008), specimen from MAY-044: A = prodorsum, dorsal view, $\mathrm{B}=$ prodorsum, lateral view, $\mathrm{C}=$ opisthosoma, lateral view, $\mathrm{D}=$ mentum of subcapitulum, $\mathrm{E}=$ genitoaggenital and anoadanal plates, $\mathrm{F}=$ trochanter and femur of leg I, G = tibia of leg IV. 
Ventral region. Setae $h$ of mentum minuscule. Formula of genital setae: $6(4+2): 3$, setae $g_{6-9}$ longer than setae $g_{1-5}$. Anoadanal plates with all adanal setae longer than anal setae, setae $a d_{2}$ thick, bent distally, other setae spiniform.

Legs setation complete. Seta $d$ of femora I slightly remote from distal end of article.

Remarks - This species described from Thailand belongs to Plonaphacarus. Mahunka (2008) mistakenly placed it in the subgenus Rhacaplacarus.

\section{Steganacarus (Rhacaplacarus) brasiliensis (Pérez-Iñigo et Baggio, 1980)} (Figs 5A-F)

Protophthiracarus brasiliensis Pérez-Iñigo et Baggio, 1980

Hoplophthiracarus brasiliensis: NiedbaŁa 2004

Material examined - One specimen deposited at DATE: BR-046, Brazil, São Paulo State, São Paulo, Instituto Butantan, $50 \mathrm{~m}$ from the Museum of Microbiology, 7.XI.2002, secondary Atlantic forest, sample of wet leaf litter, leg J. Starý.

Measurements of one specimen - Prodorsum: length 278, width 177, height 96, sensillus 68, length of prodorsal setae: interlamellar (in) 63, lamellar (le) 30, rostral (ro) 20, exobothridial (ex) 15; notogaster: length 419 , width 283, height 247 , length of notogastral setae: $c_{1}, h_{1}$ and $p s_{1} 68$; genitoaggenital plate $91 \times 81$, anoadanal plate $185 \times 88$.

Remarks - Specimens from São Paulo are slightly bigger than holotype. One specimen has spiniform $c_{1}$ seta on right side, rough, but not distally obtuse and ciliate as in holotype. Moreover vestigial setae $f_{1}$ are situated posteriorly of setae $h_{1}$. This species was erroneously classified to the genus Hoplophthiracarus (Niedbata 2004).

Distribution - Species up to the present still is known only from Brazil (Niedbata 2004).

\section{Austrophthiracarus diazae (Ojeda, 1985) \\ (Figs 6A-I)}

Calyptophthiracarus cucundus Niedbała, 1988: NiedbaŁa 2004

Material examined - Specimen no 1 is deposited in DATE, specimen no 2 at ISB and specimen no 3 at NHMG, all from the locality COL-009, Colombia, Meta, Carimagua, 15.IV.1983, native pasture, savannas, hand sampling, leg J. M. Guerrero.

Measurements of specimen no 1 - Prodorsum: length 263, width 192, height 91, sensillus 28, length of prodorsal setae: interlamellar (in) 41, lamellar (le) 23, rostral (ro) 35, exobothridial (ex) 13; notogaster: length 500 , width 323 , height 318 , length of notogastral setae: $c_{1} 58, h_{1} 51, p s_{1} 48$; genitoaggenital plate $139 \times 114$, anoadanal plate $202 \times 109$. 


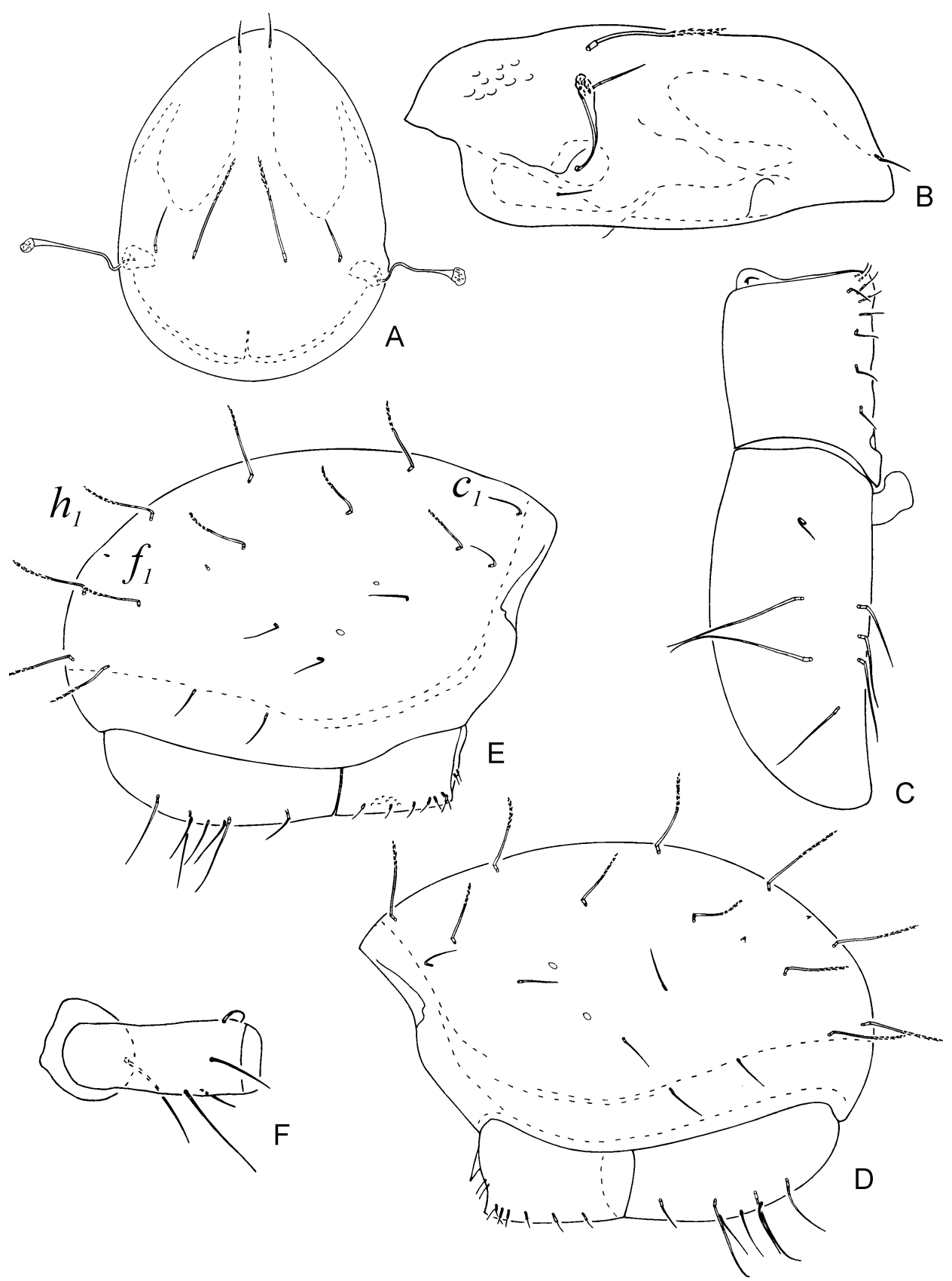

Figs 5A-F. Steganacarus (Rhacaplacarus) brasiliensis (Pérez-Iñigo et Baggio, 1980): A = prodorsum, dorsal view, $\mathrm{B}=$ prodorsum, lateral view, $\mathrm{C}=$ genitoaggenital and anoadanal plates, $\mathrm{D}=$ opisthosoma, lateral view, left side, $\mathrm{E}=$ opisthosoma, lateral view, right side, $\mathrm{F}=$ trochanter and femur of leg I. 


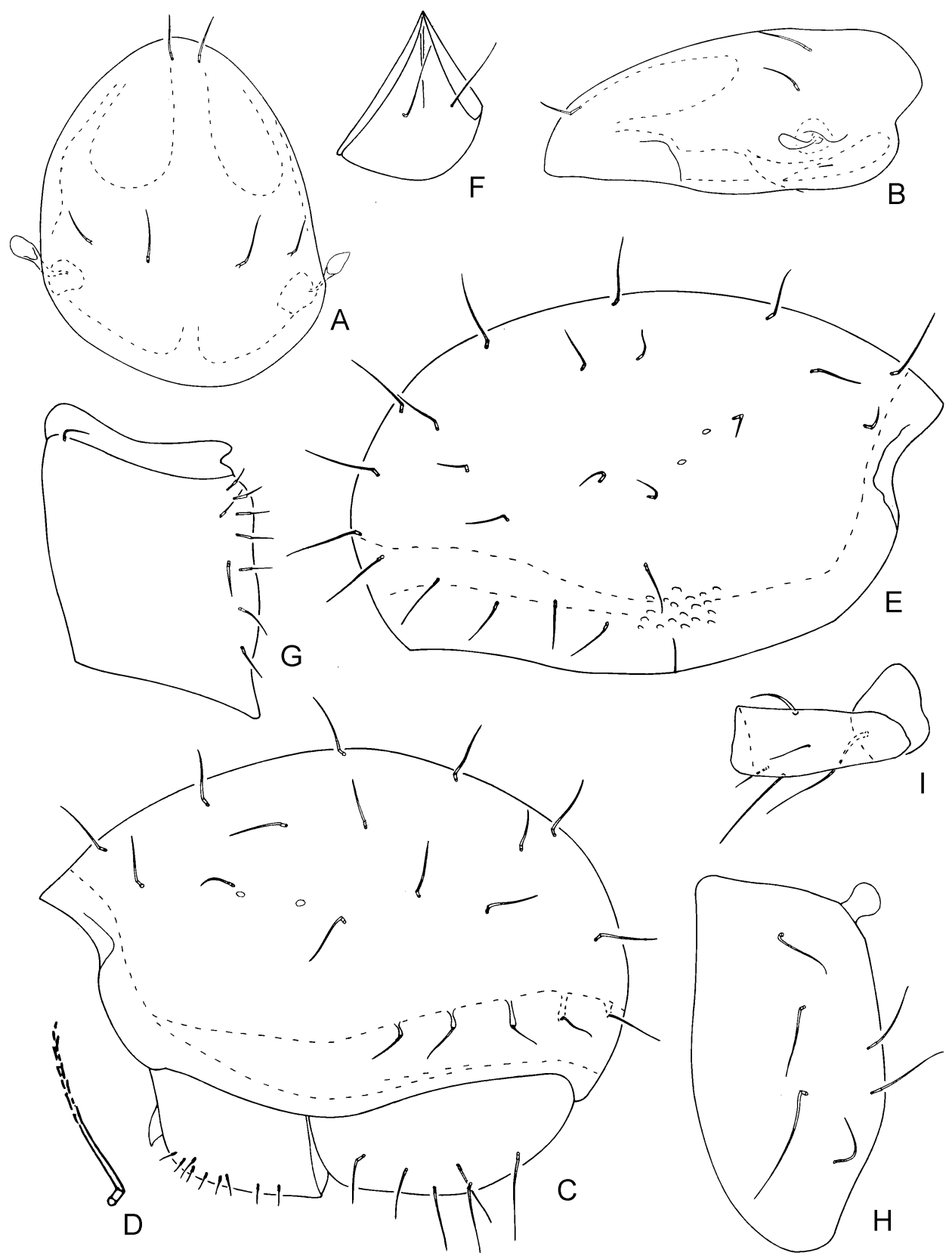

Figs 6A-I. Austrophthiracarus diazae (Ojeda, 1985): A = prodorsum, dorsal view, B = prodorsum, lateral view, $\mathrm{C}=$ opisthosoma, lateral view, $\mathrm{D}=$ seta $c_{1}, \mathrm{E}=$ notogaster, lateral view, another specimen, $\mathrm{F}=$ mentum of subcapitulum, $\mathrm{G}=$ genitoaggenital plate, $\mathrm{H}=$ anoadanal plate, I = trochanter and femur of leg I. 
Remarks - The specimens from Colombia have a different number of notogastral setae: Specimen no 1 has 19 pairs of notogastral setae, specimen no 2 has 23 pairs of setae and specimen no 3 has 23 setae on the left side and 24 setae on the right side. Specimens recorded from Venezuela (OJEDA 1985) have 20 pairs of notogastral setae, while, on the other hand, specimens from Ecuador have 19 pairs of setae (sub Calyptophthiracarus cucundus) (OJEDA 1985, NiedbaŁa 1988b, NiedbaŁa 2004).

Distribution - Neotropical species (Niedba£a 2004).

\section{Arphthicarus sculptilis (Niedbała, 1988)}

Material examined - One specimen deposited in DATE: TAN-016, Tanzania, Uluguru

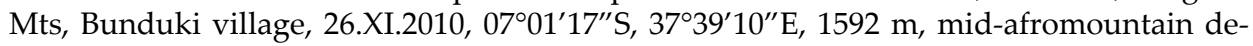
ciduous forest, litter sifting sample, leg V. Grebennikov.

Measurements of specimen from sample TAN-016 - Prodorsum: length 465, width 328 , height 151, sensillus 96, setae: interlamellar (in) 271, lamellar (le) 25, rostral (ro) 298, exobothridial (ex) 6; notogaster: length 1000, width 707, height 687, length of notogastral setae: $c_{1} 238, c_{1} / c_{1}-d_{1}=1.0, h_{1} 409, p s_{1} 368$; genitoaggenital plate $273 \times 182$, anoadanal plate $333 \times 192$.

Remarks - Notogastral setae covered with small cilia, distally obtuse, setae $h_{1}$ and $p s_{1}$ longer than other setae. Distance between setae $p s_{3}$ and $p s_{4}$ considerably longer than between $p s_{2}-p s_{3}$; vestigial setae $f_{1}$ slightly anterior of setae $h_{1}$. Shape of adanal setae $a d_{1}$ as notogastral setae, covered with small cilia, obtuse, remaining setae of anoadanal plates spiniform and rough.

Distribution - Species up to the present known from eastern islands of Afrotropical Region: Comoro Islands, Reunion, Madagascar (NiedbaŁa 1988a, 2001).

\section{Notophthiracarus lienhardi Mahunka, 1996}

$$
\text { (Figs 7A-G) }
$$

Material examined - One specimen deposited at DATE from the locality: MAY-002, Malaysia, Pahang, Cameron Highlands, Orange Asli village environs, Gunung Perdah Mt., 2.V.-14.V.2009, $04^{\circ} 29^{\prime} 0^{\prime \prime} \mathrm{N}, 101^{\circ} 22^{\prime} 06^{\prime \prime} \mathrm{E}, 1575 \mathrm{~m}$, sifting of leaf litter in shallow ravine, leg P. Baňař, two specimens deposited at DATE from the locality: MAY-003, Malaysia, Pahang, Cameron Highlands, Tanan Rata village environs, Gunung Jasar Mt., 4.V.2009, 04²8 '28' N, $101^{\circ} 21^{\prime} 36^{\prime \prime}-22^{\prime} 06^{\prime \prime} \mathrm{E}, 1620 \mathrm{~m}$, sifting of leaf litter, leg P. Baňař, one specimen deposited at DATE from the locality: MAY-004, Malaysia, Pahang, Cameron Highlands, Tanan Rata village environs, near Parit Fall, 25.IV-15.V.2009, 04 28'24" $-28^{\prime} 42^{\prime \prime} \mathrm{N}, 101^{\circ} 21^{\prime} 36^{\prime \prime}-22^{\prime} 06^{\prime \prime} \mathrm{E}$, 1470-1550 m, sifting of leaf litter and rotten wood, leg P. Baňař, three specimens from the same locality deposited at ISB.

Measurements of specimen from sample MAY-003 - Prodorsum: length 444, width 308 , height 252, sensillus 101, length of prodorsal setae: interlamellar (in) 38, lamellar (le) 

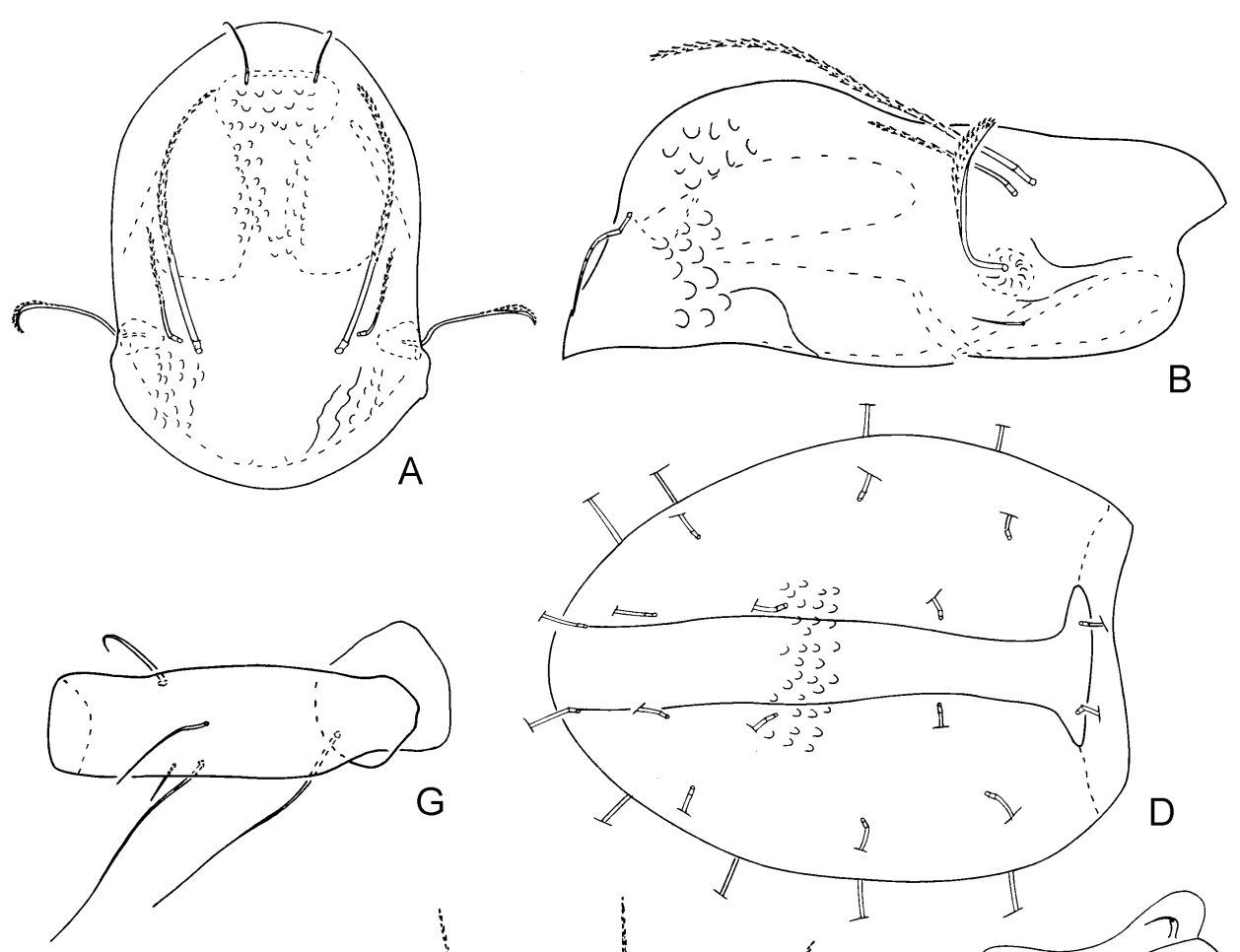

G

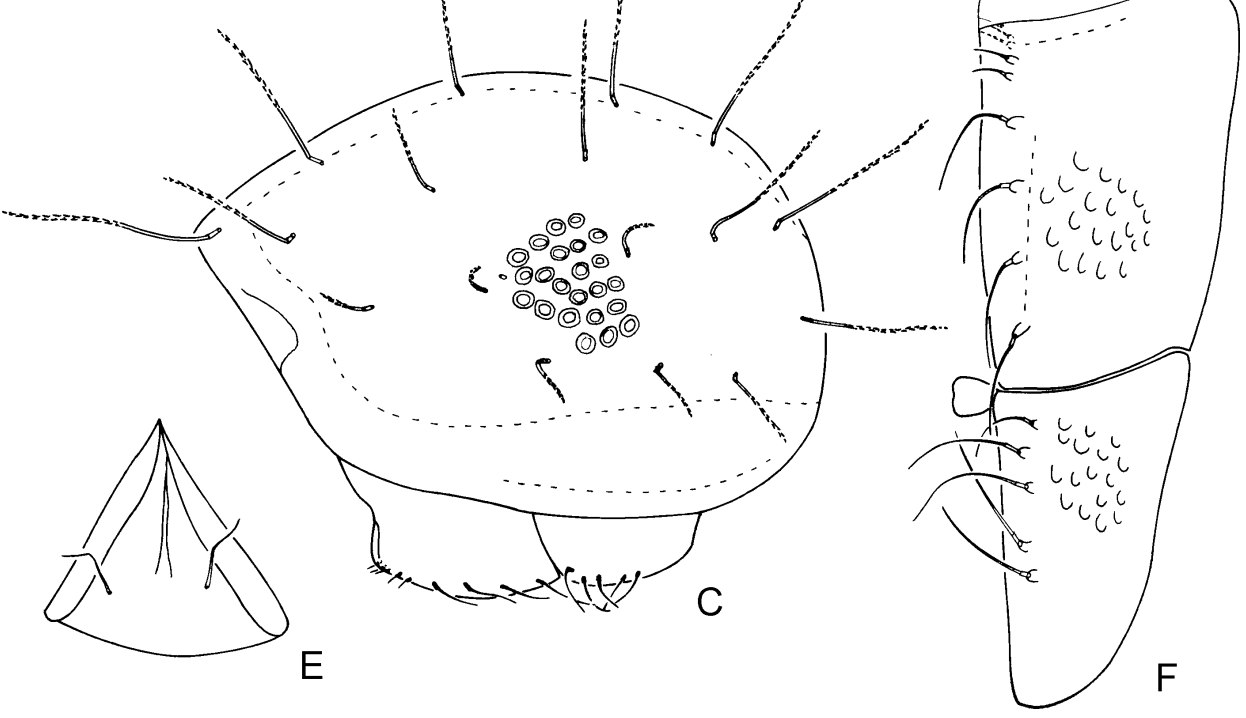

Figs 7A-G. Notophthiracarus lienhardi Mahunka, 1996: A = prodorsum, dorsal view, B = prodorsum, lateral view, $\mathrm{C}=$ opisthosoma, lateral view, $\mathrm{D}=$ notogaster, dorsal view, $\mathrm{E}=$ mentum of subcapitulum, $F=$ genitoaggenital and anoadanal plates, $G=$ trochanter and femur of leg I. 
28, rostral (ro) 33, exobothridial (ex) 23; notogaster: length 949, width and height 596, length of notogastral setae: $c_{1} 78, h_{1} 106, p s_{1}$ 86; genitoaggenital plate $190 \times 126$, anoadanal plate $164 \times 96$. The specimens now collected from Pahang are slightly larger than holotype.

Redescription - Colour dark brown. Cuticle very well sculptured, covered with foveolae, deep and well framed on notogaster.

Prodorsum with very strong median crista. Posterior furrows distinct. Sigillar fields distinct, long, median field widely dilated distally around rostral setae. Lateral carinae absent. Sensilli long, slightly sickle-like, dilated in distal half and covered with small cilia. Interlamellar (in) and lamellar (le) setae straight, erect, blunt at distal end and densely setose. Rostral setae (ro) setiform, rough and bent downwards.

Notogaster with longitudinal median band; 17 pairs of notogastral setae straight, erect, densely setose; dorsal setae longer $\left(c_{1}>c_{1}-d_{1}\right)$ than laterals. Setae $c_{1}$ situated on anterior border of notogaster, setae $c_{2}$ and $c_{3}$ slightly remote from notogastral border. Only lyrifissures $i a$ visible. Vestigial setae invisible because of strong sculpture.

Ventral region. Setae $h$ of mentum almost as long as distance between them. Genitoaggenital plates with nine pairs of genital setae with arrangement: $6: 3$, setae $g_{6-9}$ very long. Anoadanal plates each with five pairs of adanal setae also close to paraxial border but clearly separated from anal setae.

Legs. Formula of setae and solenidia of complete type. Setae $d$ of femora I considerably remote from distal end of segment, setae $v^{\prime \prime}$ more than five times longer than setae $v^{\prime}$.

Comparison $-N$. lienhardi is easy distinguishable from other Notophthiracarus species by the number of 17 pairs of notogastral setae, presence of longitudianal median band, deep and framed alveoli of notogastral surface, very long genital setae $g_{6-9}$, position of anal and adanal setae close to paraxial border of plates.

Distribution - Species up to the present known only from Malaysia ( $\mathrm{MA}^{-}$ HUNKA 1996, NiEDbAŁA 2000).

\section{Notophthiracarus stenotus Niedbała et Corpuz-Raros, 1998}

(Figs 3E-G)

Material examined - Two specimens from NG-1803, Indonesia - Irian, New Guinea, Cape Pie, near entrance of the Jountela bay, 9.I.1954, forest on coral limestone, litter sample, leg. L. van der Hammen.

Remarks - Specimens from NG-1803 have similar dimensions as the holotype but they differ in the shape of notogastral setae, which are broader and rounded. All other morphological charaters are the same as holotype (NiedbaŁa \& Corpuz-Raros 1998).

Distribution - So far the species is known only from the Philippines (NiedbaŁa 2000). 


\section{NEW RECORDS AND NEW DATA ABOUT DISTRIBUTION OF SOME SPECIES}

\section{Ptyctimous mites the first time noted from Gough Island}

Notophthiracarus fecundus Niedbała, 2000 - Material examined: GHI-03, Gough Island, southern part of the island, 16.V.1968, dense tussock, sample of grass rhizosphere, leg A. Macfadyen, (2 sp.). Distribution: Species up to the present known from New Zealand only (NiedbaŁa 2000).

Notophthiracarus minusculus Niedbała, 2004 - Material examined: GHI-03, Gough Island, southern part of the island, 16.V.1968, dense tussock, sample of grass rhizosphere, leg A. Macfadyen, (2 sp.). Distribution: Species up to the present known from Chile only (NiedbaŁa 2004).

\section{Ptyctimous mites the first time noted from Bangladesh}

Acrotritia curticephala (Jacot, 1938) - Material examined: BGL-029, Bangladesh, Khulna, Sundarbans National Park, Balirgang, 2.III.1997, 214ㄴ $13^{\prime \prime} \mathrm{N}, 89^{\circ} 21^{\prime} 66^{\prime \prime} \mathrm{E}$, mangroves, litter sample under gewa tree, leg M. A. Habib, (2 sp.), BGL-037, Bangladesh, Khulna, Sundarbans National Park, Supati forest station, 25.II.1997, 21 ${ }^{\circ} 02^{\prime} 62^{\prime \prime} \mathrm{N}, 89^{\circ} 49^{\prime} 38^{\prime \prime} \mathrm{E}$, mangroves, litter sample near pond, leg M. A. Habib, (1 sp.). Distribution: Semicosmopolitan species; not reported from Afrotropical Region (Niedbata 2004).

Phthiracarus compressus Jacot, 1930 - Material examined: BGL-003, Bangladesh,

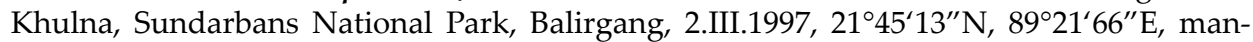
groves, sample of lichens on goran tree, leg M. A. Habib, (1 sp.). Distribution: Holarctic species (NiedbaŁa 2011).

Atropacarus (Hoplophorella) vitrinus (Berlese, 1913) - Material examined: BGL037, Bangladesh, Khulna, Sundarbans National Park, Supati forest station, 25.II.1997, $21^{\circ} 02^{\prime} 62^{\prime \prime} \mathrm{N}, 89^{\circ} 49^{\prime} 38^{\prime \prime} \mathrm{E}$, mangroves, litter sample near pond, leg M. A. Habib, (2 sp.). Distribution: Semicosmopolitan species (NiedbaŁa 2011).

\section{Ptyctimous mites the first time noted from Colombia}

Mesoplophora (Mesoplophora) parabacilla Niedbała, 2006 - Material examined: COL-009, Colombia, Meta, Carimagua, 15.IV.1983, native pasture, savannas, hand sampling, leg J. M. Guerrero, (2 sp.). Distribution: Species up to the present known from Panama only (NiedbaєA 2006).

Austrophthiracarus diazae (Ojeda, 1985) - Material examined: COL-009, Colombia, Meta, Carimagua, 15.IV.1983, native pasture, savannas, hand sampling, leg J. M. Guerrero, (3 sp.). Distribution: Neotropical species up to the present known from Costa Rica, Panama, Venezuela, Ecuador and Chile (NiedbaŁa 2004).

Notophthiracarus aculetaus Niedbała, 1988 - Material examined: COL-009, Colombia, Meta, Carimagua, 15.IV.1983, native pasture, savannas, hand sampling, leg. J. M. Guerrero, $(4$ sp.). Distribution: Species up to the present known from Panama and Ecuador (NiedbaŁa 1988b, 2004). 


\section{Ptyctimous mites the first time noted from Tanzania}

Indotritia paraconsimilis Niedbała, 2012 - Material examined: TAN-014, Tanzania,

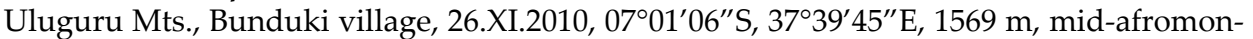
tane deciduous forest, litter sifting sample, leg. V. Grebennikov (4 sp.), TAN-016, Tanzania,

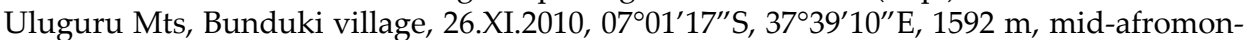
tane deciduous forest, litter sifting sample, leg. V. Grebennikov (17 sp.). Distribution: Species up to the present was known from Ethiopia only (NiedbaŁa \& Ermilov 2012).

Hoplophthiracarus ambiguus Niedbała, 1982 - Material examined: TAN-014, Tanzania, Uluguru Mts, Bunduki village, 26.XI.2010, 0701'06”S, 37³9'45”E, 1569 m, midafromontane deciduous forest, litter sifting sample, leg. V. Grebennikov (13 sp.). Distribution: Species known from central part of Afrotropical Region (NiedbaŁA 1982, 2001).

\section{Ptyctimous mites the first time noted from Brazil}

Acrotritia parallelos (Niedbała, 2003) - Material examined: BR-046, Brazil, São Paulo State, São Paulo, Instituto Butantan, 50 m from Museum of Microbiology, 7.XI.2002, secondary Atlantic forest, sample of wet leaf litter, leg. J. Starý, (1 sp.). Distribution: Neotropical endemic species up to the present known Costa Rica and Panama (NiedbaŁa 2003, 2004).

\section{Ptyctimous mites the first time noted from New Guinea (Indonesia, Irian)}

Notophthiracarus stenotus Niedbała et Corpuz-Raros, 1998 - Material examined: NG-1803, Indonesia - Irian, New Guinea, Cape Pie, near entrance of the Jountela Bay, 9.I. 1954, forest on coral limestone, litter sample, leg. L. van der Hammen (2 sp.). Distribution: Species up to the present known from the Philippines only (Niedba£a \& Corpuz-Raros 1998, Niedbaea 2000).

Acrotritia refracta (Niedbała, 1998) - Material examined: NG-1806, Indonesia - Irian, New Guinea, Korido, Loepiori, 9.IV.1954, forest on chlorite schist, litter sample, leg. L. van der Hammen, (1 sp.). Distribution: Pantropical species (Niedba£a 2004).

Atropacarus (Hoplophorella) singularis Sellnick, 1959 - Material examined: NG-1815, Indonesia - Irian, New Guinea, Woendi Padaido Islands, 1.III.1954, beach forest, litter sample, leg. L. van der Hammen, (1 sp.). Distribution: Pantropical species (NiedbaŁa 2004).

\section{Ptyctimous mites the first time noted from Malaysia}

Oribotritia lepteces Niedbała, Corpuz-Raros et Gruezo, 2006 - Material examined: MAY-001, Malaysia, Pahang, Cameron Highlands, Tanan Rata village environs, near Parit

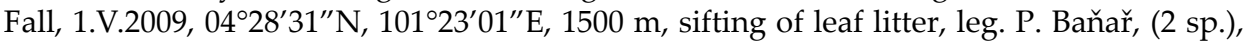
MAY-003, Malaysia, Pahang, Cameron Highlands, Gunung Jasar Mt., Tanan Rata village environs, 4.V.2009, $04^{\circ} 28.47^{\prime} \mathrm{N}, 101^{\circ} 21^{\prime} 36^{\prime \prime}-22^{\prime} 06^{\prime \prime} \mathrm{E}, 1620 \mathrm{~m}$, sifting of leaf litter, leg. P. Baňař, (1 sp.). Distribution: Species up to the present known from the Philippines and New South Wales, Australia, (NiedbaŁa et al. 2006).

Oribotritia nepalensis Niedbała, 2000 - Material examined: MAY-003, Malaysia, Pahang, Cameron Highlands, Gunung Jasar Mt., Tanan Rata village environs, 4.V.2009, $04^{\circ} 28^{\prime} 28^{\prime \prime} \mathrm{N}, 1^{\circ} 21^{\prime} 36^{\prime \prime}-22^{\prime} 06^{\prime \prime} \mathrm{E}, 1620 \mathrm{~m}$, sifting of leaf litter, leg. P. Baňař, (1 sp.). Distribution: Species up to the present known from Nepal and Tibet (NiedbaŁa 2000). 
Austrotritia robusta Niedbała et Corpuz-Raros, 1998 - Material examined: MAY-003, Malaysia, Pahang, Cameron Highlands, Gunung Jasar Mt., Tanan Rata village environs, 4. V.2009, $04^{\circ} 28^{\prime} 28^{\prime \prime} \mathrm{N}, 101^{\circ} 21^{\prime} 36^{\prime \prime}-22^{\prime} 06^{\prime \prime} \mathrm{E}, 1620 \mathrm{~m}$, sifting of leaf litter, leg. P. Baňař, (3 sp.). Distribution: Pantropical species (NiedBa€A 2000).

Plonaphacarus semiaciculatus (Mahunka, 2008) - Material examined: MAY-044, West Malaysia, Batu Caves, 30.XII.2010, $03^{\circ} 14^{\prime} 50^{\prime \prime} \mathrm{N}, 101^{\circ} 41^{\prime} 17^{\prime \prime} \mathrm{E}$, rain forest on limestone bedrock, wet site with rich brushwood, sample of leaf litter, leg. J. Farská, (3 sp.). Distribution: Species up to the present known from Thailand only (MAнUnKa 2008).

\section{Ptyctimous mites the first time noted from Reunion}

Phthiracarus nitens (Nicolet, 1855) - Material examined: REU-007, Reunion, volcano Cirque de Cillaos, 16.XI.2007, old coniferous forest, sample of mosses and soil, leg. M. Veselovský, (1 sp.). Distribution: Western Palaearctic species introduced to Reunion (NiedbaŁa 2011).

\section{Ptyctimous mites the first time noted from Jamaica}

Acrotritia refracta (Niedbała, 1998) - Material examined: JAM-46, Jamaica, Blue Mountains, near waterfall on the Shentamee river, about $15 \mathrm{~km}$, north of Kingston, 18.VI. 1992, dense montane foggy forest, moss sample, leg. R. Hogervorst, (1 sp.). Distribution: Pantropical species (NiedbaŁa 2004).

Phthiracarus anonymus Grandjean, 1933 - Material examined: JAM-44, Jamaica, Blue Mountains, near waterfall on Shentamee river, about $15 \mathrm{~km}$, north of Kingston, 16.VI. 1993, dense montane foggy forest, sample of leaf litter, leg. R. Hogervorst, (2 sp.). Distribution: Semicosmopolitan species (Niedba£a 2011).

Arphthicarus latebrosus (Niedbała, 1982) - Material examined: JAM-45, Jamaica, Blue Mountains, near waterfall on Shentamee river, about $15 \mathrm{~km}$, north of Kingston, 17.VI. 1993, montane deciduous forest, rotten wood sample, leg. R. Hogervorst, (1 sp.). Distribution: Pan-neotropical species (Niedbata 2004).

\section{Ptyctimous mites the first time noted from Cuba}

Indotritia bellingeri Niedbała et Schatz, 1996 - Material examined: K-153, Cuba, Province Guantanamo, Guantanamo, garden of the Instituto del Suelos, 17.XI.1979, soil sample, leg. J. Rusek, (1 sp.), K-322, Cuba, Province Habana, Arroyo Bermejo, 25.X.1981, semideciduous forest, leaf litter sample, leg. J. Rusek, (1 sp.). Distribution: Pan-neotropical species (NiedbaŁa 2004).

Indotritia krakatauensis (Sellnick, 1923) - Material examined: K-141, Cuba, Province Pinar del Rio, Guanahacabites, sea shore forest, sandy soil sample, leg. J. Rusek, (3 sp.), K-148, Cuba, Province Pinar del Rio, Guanahacabites, 10.XI.1979, sea shore forest, sandy soil sample, leg. J. Rusek, (1 sp.), K-152, Cuba, Province Guantanamo, garden of the Instituto del Suelos, 17.XI.1979, soil sample, leg. J. Rusek, (2 sp.). Distribution: Pantropical species (Niedbata 2004).

Microtritia tropica Märkel, 1964 - Material examined: K-148, Cuba, Province Pinar del Rio, Guanahacabites, 10.XI.1979, sea shore forest, sandy soil sample, leg. J. Rusek, (1 sp.). Distribution: Pantropical species (NiedbaŁa 2004). 
Ptyctimous mites the first time noted from the USA

Oribotritia paracarolinae Niedbała, 2007 - Material examined: USA-305, Washington, Pierce County, south of Forest Lake, 20.I.1991, $47^{\circ} 02^{\prime} 42^{\prime \prime} \mathrm{N}, 122^{\circ} 11^{\prime} 31^{\prime \prime} \mathrm{W}, 170 \mathrm{~m}$, sample of Alnus rubra, Salix sp. litter, leg. R. Crawford, (1 sp.), USA-308, Washington, Pierce County, Maplewood Spring, 20.IV.1992, 47 $10^{\circ} 30^{\prime \prime} \mathrm{N}, 122^{\circ} 18^{\prime} 58^{\prime \prime} \mathrm{W}, 15 \mathrm{~m}$, deciduous forest, Acer macrophyllum litter sample, leg. R. Crawford (3 sp.). Distribution: Species up to the present known from Canada only (NiedbaŁa 2007).

\section{Ptyctimous mites the first time noted from Canada}

Euphthiracarus tanythrix Walker, 1965 - Material examined: CAN-107, Canada, Ontario, Moosonee, 3.X.1983, spruce forest, litter and soil sample, leg. J. Tomlin, (1 sp.). Distribution: Species up to the present known from the USA (Walker 1965, Niedbata 2002).

\section{NEW LOCALITIES OF SOME KNOWN SPECIES}

\section{Records from Indonesia}

Austrotritia lebronneci (Jacot, 1934) - Material examined: NG-1806, Indonesia Irian, New Guinea, Korido, Loepiori, 9.IV.1954, forest on chlorite schist, leaf litter sample, leg. L. van der Hammen, (1 sp.). Distribution: Pantropical species (NiedbaŁa 2000).

\section{Records from Malaysia}

Apoplophora ornata Niedbała, 2000 - Material examined: MAY-044, West Malaysia, Batu Caves, 30.XII.2010, $03^{\circ} 14^{\prime} 50^{\prime \prime} \mathrm{N}, 101^{\circ} 41^{\prime} 17^{\prime \prime} \mathrm{E}$, rain forest on limestone bedrock, wet site with rich brushwood, sample of leaf litter, leg. J. Farská, (30 sp.). Distribution: Species up to the present known from Borneo only (Niedbata 2000).

Apoplophora pantotrema (Berlese, 1913) - Material examined: MAY-002, Malaysia, Pahang, Cameron Highlands, Gunung Perdah Mt., Orange Asli village environs, 2-14. V.2009, $04^{\circ} 29^{\prime} 01^{\prime \prime} \mathrm{N}, 101^{\circ} 22^{\prime} 06^{\prime \prime} \mathrm{E}, 1575 \mathrm{~m}$, sifting of leaf litter in shallow ravine, leg. P. Baňař, (1 sp.); MAY-003, Malaysia, Pahang, Cameron Highlands, Gunung Jasar Mt., Tanan Rata village environs, 4.V.2009, 04 ${ }^{\circ} 8^{\prime} 28^{\prime \prime} \mathrm{N}, 101^{\circ} 21^{\prime} 36^{\prime \prime}-22^{\prime} 06^{\prime \prime} \mathrm{E}, 1620 \mathrm{~m}$, sifting of leaf litter, leg. P. Baňař, (1 sp.); MAY-004, Malaysia, Pahang, Cameron Highlands, Tanan Rata village environs, near Parit Fall, 25.IV-15.V.2009, 04 28 $24^{\prime \prime}-28^{\prime} 42^{\prime \prime} \mathrm{N}, 101^{\circ} 21^{\prime} 36^{\prime \prime}-22^{\prime} 06^{\prime \prime} \mathrm{E}$, 1470-1550 m, sifting of leaf litter and rotten wood, leg. P. Baňař, (1 sp.); MAY-044, West Malaysia, Batu Caves, 30.XII.2010, 03 ${ }^{\circ} 14^{\prime} 50^{\prime \prime} \mathrm{N}, 101^{\circ} 41^{\prime} 17^{\prime \prime} \mathrm{E}$, rain forest on limestone bedrock, wet site with rich brushwood, sample of leaf litter, leg. J. Farská (3 sp.). Distribution: Oriental species introduced to Palaearctic (Japan), Australian (Papua New Guinea and Queensland) and the West Pacific islands (Solomon and Fiji) Regions (Niedbata 2000).

Mesoplophora (Parplophora) paraleviseta Mahunka, 1991 - Material examined: MAY-044, West Malaysia, Batu Caves, 30.XII.2010, 03¹4'50”N, 101 ${ }^{\circ} 41^{\prime} 17^{\prime \prime} \mathrm{E}$, rain forest on limestone bedrock, wet site with rich brushwood, sample of leaf litter, leg. J. Farská, (1 sp.). Distribution: Species up to the present known from Malaysia only, perhaps endemic (NiedbaŁa 2000). 
Acrotritia ardua (C. L. Koch, 1941) - Material examined: MAY-044, West Malaysia, Batu Caves, 30.XII.2010, 03 ${ }^{\circ} 14^{\prime} 50^{\prime \prime} \mathrm{N}, 101^{\circ} 41^{\prime} 17^{\prime \prime} \mathrm{E}$, rain forest on limestone bedrock, wet site with rich brushwood, sample of leaf litter, leg. J. Farská, (1 sp.). Distribution: Semicosmopolitan species (NiedbaŁa 2011).

Acrotritia curticephala (Jacot, 1938) - Material examined: MAY-044, West Malaysia, Batu Caves, 30.XII.2010, 03 $14^{\prime} 50^{\prime \prime} \mathrm{N}, 101^{\circ} 41^{\prime} 17^{\prime \prime} \mathrm{E}$, rain forest on limestone bedrock, wet site with rich brushwood, sample of leaf litter, leg. J. Farská, (1 sp.). Distribution: Semicosmopolitan species not reported from Afrotropical Region (NiEdBAŁA 2004).

Oribotritia aokii Mahunka, 1987 - Material examined: MAY-003, Malaysia, Pahang, Cameron Highlands, Gunung Jasar Mt., Tanan Rata village environs, 4.V.2009, 04 $28^{\prime} 28^{\prime \prime} \mathrm{N}$, $101^{\circ} 21^{\prime} 36^{\prime \prime}-22^{\prime} 06^{\prime \prime} \mathrm{E}, 1620 \mathrm{~m}$, sifting of leaf litter, leg. P. Baňař, (1 sp.). Distribution: Oriental species (NiedbaŁa, W. \& Corpuz-Raros 1998, NiedbaŁa 2000).

Oribotritia bulbifer (Mahunka, 1987) - Material examined: MAY-044, West Malaysia, Batu Caves, 30.XII.2010, 03 ${ }^{\circ} 14^{\prime} 50^{\prime \prime} \mathrm{N}, 101^{\circ} 41^{\prime} 17^{\prime \prime} \mathrm{E}$, rain forest on limestone bedrock, wet site with rich brushwood, sample of leaf litter, leg. J. Farská, (1 sp.). Distribution: Oriental species (NiedbaŁa 2000).

Indotritia javensis (Sellnick, 1923) - Material examined: MAY-002, Malaysia, Pahang, Cameron Highlands, Gunung Perdah Mt., Orange Asli village environs, 2-14.V. $2009,04^{\circ} 29^{\prime} 01^{\prime \prime} \mathrm{N}, 101^{\circ} 22^{\prime} 06^{\prime \prime} \mathrm{E}, 1575 \mathrm{~m}$, sifting of leaf litter in shallow ravine, leg. P. Baňař, (5 sp.); MAY-003, Malaysia, Pahang, Cameron Highlands, Gunung Jasar Mt., Tanan Rata village environs, $4 . \mathrm{V} .2009,04^{\circ} 28^{\prime} 28^{\prime \prime} \mathrm{N}, 101^{\circ} 21^{\prime} 36^{\prime \prime}-22^{\prime} 06^{\prime \prime} \mathrm{E}, 1620 \mathrm{~m}$, sifting of leaf litter, leg. P. Baňař, (5 sp.); MAY-004, Malaysia, Pahang, Cameron Highlands, near Parit Fall, Tanan Rata village environs, 25.IV.-15.V.2009, 04²8 $24^{\prime \prime}-28^{\prime} 42^{\prime \prime} \mathrm{N}, 1^{\circ} 1^{\circ} 21^{\prime} 36^{\prime \prime}-22^{\prime} 06^{\prime \prime} \mathrm{E}$, ca 1470-1550 m, sifting of leaf litter and rotten wood, leg. P. Baňař. (16 sp.). Distribution: Oriental species introduced in the border zone of the Palaearctic Region (NiedBA£A 2000).

Plonaphacarus kugohi (Aoki, 1959) - Material examined: MAY-043, West Malaysia, Batu Caves, 30.XII.2010, 03 ${ }^{\circ} 14^{\prime} 50^{\prime \prime} \mathrm{N}, 101^{\circ} 41^{\prime} 17^{\prime \prime} \mathrm{E}$, rain forest on limestone bedrock, wet site with rich brushwood, sample of litter and soil, leg. J. Farská, (4 sp.); MAY-044, West Malaysia, Batu Caves, 30.XII.2010, 0314'50”N , $101^{\circ} 41^{\prime} 17^{\prime \prime} \mathrm{E}$, rain forest on limestone bedrock, wet site with rich brushwood, sample of leaf litter, leg. J. Farská, (3 sp.). Distribution: Pantropical species (NiedbaŁa 2000).

Notophthiracarus lienhardi Mahunka, 1996 - Material examined: MAY-002, Malaysia, Pahang, Cameron Highlands, Gunung Perdah Mt., Orange Asli village environs, 2.V. $-14 . V .2009,04^{\circ} 29^{\prime} 12^{\prime \prime} \mathrm{N}, 101^{\circ} 22^{\prime} 06^{\prime \prime} \mathrm{E}, 1575 \mathrm{~m}$, sifting of leaf litter in shallow ravine, leg. P. Baňař, (1 sp.); MAY-003, Malaysia, Pahang, Cameron Highlands, Gunung Jasar Mt., Tanan Rata village environs, 4.V.2009, $04^{\circ} 28^{\prime} 28^{\prime \prime} \mathrm{N}, 101^{\circ} 21^{\prime} 36^{\prime \prime}-22^{\prime} 06^{\prime \prime} \mathrm{E}, 1620 \mathrm{~m}$, sifting sample of leaf litter, leg. P. Baňař, (2 sp.); MAY-004, Malaysia, Pahang, Cameron Highlands, Tanan Rata village environs, near Parit Fall, 25.IV-15.V.2009, 04 $28^{\prime} 24^{\prime \prime}-28^{\prime} 42^{\prime \prime} \mathrm{N}, 1^{\circ} 1^{\circ} 21^{\prime} 36^{\prime \prime}-$ 22.'06" E, 1470-1550 m, sifting of leaf litter and rotten wood, leg. P. Baňař, (4 sp.). Distribution: Species up to the present known from Malaysia, perhaps endemic (NiedbaŁA 2000).

Atropacarus (Hoplophorella) cucullatus (Ewing, 1909) - Material examined: MAY044, West Malaysia, Batu Caves, 30.XII.2010, 03¹4'50”N, 101 ${ }^{\circ} 41^{\prime} 17^{\prime \prime}$ E, rain forest on limestone bedrock, wet site with rich brushwood, sample of leaf litter, leg. J. Farská, (1 sp.). Distribution: Semicosmopolitan species (NiedbaŁa 2000).

Atropacarus (Hoplophorella) vitrinus (Berlese, 1913) - Material examined: MAY-043, West Malaysia, Batu Caves, 30.XII.2010, 03 ${ }^{\circ} 14^{\prime} 50^{\prime \prime} \mathrm{N}, 101^{\circ} 41^{\prime} 17^{\prime \prime} \mathrm{E}$, rain forest on limestone bedrock, wet site with rich brushwood, sample of litter and soil, leg. J. Farská, (1 sp.). Distribution: Pantropical species (NiedbaŁa 2000). 


\section{Records from Reunion}

Indotritia krakatauensis (Sellnick, 1923) - Material examined: REU-001, Reunion, volcano Piton de la Fournaise, near tourist trail, 19.XI.2007, ca 2000 m, dense bush growth, sample of grass rhizosphere, leg. M. Veselovský, (1 sp.). Distribution: Pantropical species (Niedbata 2004).

Notophthiracarus parareductus Mahunka, 1992 - Material examined: REU-007, Reunion, volcano Cirque de Cillaos, 16.XI.2007, old coniferous forest, sample of mosses and soil, leg. M. Veselovský, (1 sp.). Distribution: Species up to the present known from Reunion, perhaps endemic (NiedbaŁa 2001).

\section{Records from the Seychelles}

Mesoplophora (Parplophora) leviseta Hammer, 1979 - Material examined: SEY-001, Seychelles, Praslin, Valle de Mai, 12.XII.1975, forest with Lodricea sp., sample of dry soil and litter, leg. A. Fjellberg, (8 sp.); SEY-004, Seychelles, Mahé, Morne, 3.XII.1975, 850 m, deciduous forest, leaf litter sample, leg. A. Fjellberg, $(9 \mathrm{sp}$.$) . Distribution: Oriental species$ introduced to Seychelles (NiedbaŁa 2010)

Indotritia krakatauensis (Sellnick, 1923) - Material examined: SEY-003, Seychelles, Mahé, Tahamaku, 6.XII.1975, swamps in coconut plantation, litter sample, leg. A. Fjellberg, (2 sp.). Distribution: Pantropical species (NiedbaŁa 2004, 2010).

Microtritia tropica Märkel, 1964 - Material examined: SEY-002, Seychelles, Mahé, Casqdore, 10.XII.1975, palm and bamboo forest, leaf litter sample, leg. A. Fjellberg, SEY005, Seychelles, Mahé, Morne, 3.XII.1975, 750 m, deciduous forest, leaf litter sample, leg. A. Fjellberg, (13 sp.). Distribution: Pantropical species (Niedba€A 2010).

Phthiracarus schauenbergi (Mahunka, 1988) - Material examined: SEY-005, Seychelles, Mahé, Morne, 3.XII.1975, $750 \mathrm{~m}$, deciduous forest, leaf litter sample, leg. A. Fjellberg, (3 sp.). Distribution: Afrotropical species (NiedbaŁa 2010).

\section{Records from Kenya}

Acrotritia ardua (C. L. Koch, 1841) - Material examined: KN-007, Kenya, Malindi, $100 \mathrm{~km}$ south of Mombasa, $500 \mathrm{~m}$ from Indian Ocean coast, 12.III.1974, sample of baobab leaf litter, leg. J. Michejda, (2 sp.). Distribution: Semicosmopolitan species (NiEdba£A 2011).

\section{Records from Canada}

Mesotritia flagelliformis (Ewing, 1909) - Material examined: CAN-101, Canada, Moonsonee, 3.X.1983, spruce forest, spruce litter and soil sample, leg. J. Tomlin, (1 sp.). Distribution: Holarctic species (NiedbaŁa 2002).

Protoribotritia canadaris Jacot, 1938 - Material examined: CAN-114, Canada, Ontario, Moosonee, 3.X.1983, spruce forest, spruce litter and soil sample, leg. J. Tomlin, (1 sp.). Distribution: Nearctic species restricted to northern and eastern parts of the Nearctic Region with southern disjunction to the New Mexico (Niedbata 2002).

Euphthiracarus cernuus Walker, 1965 - Material examined: C-72, Canada, Vancouver Island, Mac Millan Park, west of Parksville, 12.X.1974, Douglas fir old forest, sample of 
decaying wood, leg. J. Rusek, (2 sp.). Distribution: Nearctic species restricted to the west of the Nearctic Region (NiedbaŁa 2002).

Euphthiracarus flavus (Ewing, 1909) - Material examined: CAN-114, Canada, Ontario, Moosonee, 3.X.1983, spruce forest, spruce litter and soil sample, leg. J. Tomlin, (2 sp.). Distribution: Nearctic species widely distributed but absent from southwest of the Nearctic Region (NiedbaŁa 2002).

Euphthiracarus pulchrus Jacot, 1930 - Material examined: C-72, Canada, Vancouver Island, Mac Millan Park, west of Parksville, 12.X.1974, Douglas fir old forest, sample of decaying wood, leg. J. Rusek, (4 sp.); C-91, Canada, Coast Mountains, Garibaldi Provincial Park, Paul Ridge, 16.X.1974, Tsuga mertensiana forest, hygric site, sample of rotten wood, leg. J. Rusek, (1 sp.), C-185, Canada, British Columbia, Vancouver Island, China Beech Park, 8.IV.1975, Tsuga heterophylla and Picea sitchensis growth, sample of decaying wood, leg. J. Rusek, (1 sp.). Distribution: Nearctic species so far restricted to east of the Nearctic Region (Niedbaea 2002).

Acrotritia ardua (C. L. Koch, 1841) - Material examined: CAN-113, Canada, Ontario, Moonsonee, 3.X.1983, spruce forest, litter and soil sample, leg. J. Tomlin, (2 sp.); CAN-119 Canada, Ontario, Moonsonee, Tamarack, 12.V.1990, spruce forest, lichen sample on the ground, leg. J. Tomlin, (20 sp.); CAN-121, Canada, Ontario, Moonsonee, 27.VI.1990, black spruce forest, spruce litter sample, leg. J. Tomlin, (2 sp.); CAN-122 Canada, Ontario, Moonsonee, Tamarack, 12.V.1990, wet Sphagnum sp. sample, leg. J. Tomlin, (4 sp.); CAN-124, Canada, Ontario, Moonsonee, Kinosheoe Tower, 25.VI.1990, spruce forest, lichen sample on the ground, leg. J. Tomlin, (4 sp.). Distribution: Semicosmopolitan species (NiedbaŁA 2011).

Microtritia simplex (Jacot, 1930) - Material examined: CAN-114, Canada, Ontario, Moosonee, 3.X.1983, spruce forest, spruce litter and soil sample, leg. J. Tomlin, (2 sp.); C-183, Canada, Vancouver Island, China Beach Park, on west coast, 8.IV.1975, Tsuga heterophylla forest, sample of decaying wood, leg. J. Rusek, (1 sp.). Distribution: Nearctic species (Niedbata 2002).

Phthiracarus boresetosus Jacot, 1930 - Material examined: CAN-121, Canada, Ontario, Moonsonee, 27.VI.1990, black spruce forest, spruce litter sample, leg. J. Tomlin, (2 sp.). Distribution: Semicosmopolitan species (Niedba£A 2002, 2011).

Phthiracarus brevisetae Jacot, 1930 - Material examined: CAN-119, Canada, Ontario, Moonsonee, Tamarack, 12.V.1990, spruce forest, lichen sample on the ground, leg. J. Tomlin, (3 sp.). Distribution: Nearctic species (NiedbaŁa 2002).

Phthiracarus longulus (C. L. Koch, 1841) - Material examined: CAN-103, Canada, Moonsonee, 3.X.1983, spruce forest, spruce litter and soil sample, leg. J. Tomlin, (1 sp.); CAN-113, Canada, Ontario, Moonsonee, 3.X.1983, spruce forest, litter and soil sample, leg. J. Tomlin; CAN-114, Canada, Ontario, Moosonee, 3.X.1983, spruce forest, spruce litter and soil sample, leg. J. Tomlin, (1 sp.); CAN-121, Canada, Ontario, Moonsonee, 27.VI.1990, black spruce forest, spruce litter sample, leg. J. Tomlin, (3 sp.). Distribution: Holarctic species (NiedbaŁa 2002, 2011).

Phthiracarus modestus Niedbała, 1988 - Material examined: CAN-122, Canada, Ontario, Moonsonee, Tamarack, 12.V.1990, wet Sphagnum sp. sample, leg. J. Tomlin, (1 sp.). Distribution: Nearctic species restricted so far to west of the Nearctic Region (NiedbaŁA 2002).

Phthiracarus nitidus Niedbała, 1986 - Material examined: CAN-120, Canada, Ontario, Moonsonee County, 24.VI.1990, river shore, coastal fern rhizosphere, mosses, sedge rhizosphere sample, leg. J. Tomlin, (2 sp.). Distribution: Nearctic species (Niedba€a 2002). 
Phthiracarus validus Niedbała, 1986 - Material examined: CAN-112, Canada, Moonsonee, 3.X.1983, spruce forest, spruce litter and soil sample, leg. J. Tomlin, (2 sp.). Distribution: Nearctic species (Niedba€A 2002).

Hoplophthiracarus illinoisensis (Ewing, 1909) - Material examined: CAN-120, Canada, Ontario, Moonsonee County, 24.VI.1990, river shore, coastal fern rhizosphere, mosses, and sedge rhizosphere sample, leg. J. Tomlin, (7 sp.); CAN-122 Canada, Ontario, Moonsonee, Tamarack, 12.V.1990, wet Sphagnum sp. sample, leg. J. Tomlin, (2 sp.). Distribution: Holarctic species (NiedbaєA 2002, 2011).

Steganacarus (Rhacaplacarus) thoreaui (Jacot, 1930) - Material examined: CAN-111, Canada, Moonsonee, 3.X.1983, spruce forest, spruce litter and soil sample, leg. J. Tomlin, (1 sp.); CAN-121, Canada, Ontario, Moonsonee, 27.VI.1990, black spruce forest, spruce litter sample, leg. J. Tomlin; CAN-124, Canada, Ontario, Moonsonee, Kinosheoe Tower, 25.VI. 1990, spruce forest, lichen sample on the ground, leg. J. Tomlin, (1 sp.). Distribution: Nearctic species absent so far from the west of the Nearctic Region (NiedbaŁa 2002).

Atropacarus (Atropacarus) striculus (C. L. Koch, 1835) - Material examined: CAN-113, Canada, Ontario, Moonsonee, 3.X.1983, spruce forest, litter and soil sample, leg. J. Tomlin, (4 sp.); CAN-118 Canada, Ontario, Moonsonee, North Point, 27.VI.1990, coastal marsh, pitfall trap, leg. J. Tomlin, (8 sp.); CAN-119, Canada, Ontario, Moonsonee, Tamarack, 12.V.1990, spruce forest, lichen sample on the ground, leg. J. Tomlin, (2 sp.); CAN-123, Canada, Ontario, Moonsonee, 24.VI.1990, spruce forest, fen litter and moss sample, leg. J. Tomlin, (9 sp.); CAN125, Canada, Ontario, Moonsonee 7.VII.1984, spruce forest, spruce litter and soil sample, leg. J. Tomlin, (1 sp.). Distribution: Holarctic species (NiedbaŁa 2002, 2011).

\section{Records from the USA}

Archoplophora rostralis (Willmann, 1930) - Material examined: USA-73, Pennsylva-

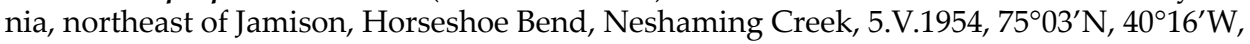
litter and soil sample, leg. W. Ivie, (8 sp.); USA-310, Minnesota, Chippewa National Forest, Leech Lake, Ottertail, 26.IX.2010, $47^{\circ} 15^{\prime} 59^{\prime \prime} \mathrm{N}, 9^{\circ} 23^{\prime} 37^{\prime \prime} \mathrm{W}, 449 \mathrm{~m}$, deciduous forest with dominant sugar maple, leaf litter sample, leg. J. Schlaghamerský, (5 sp.). Distribution: Semicosmopolitan species (NiedbaŁa 2002).

Oribotritia banksi (Oudemans, 1916) - Material examined: USA-77, Pennsylvania, northeast of Jamison, Horseshoe Bend, Neshaming Creek, $75^{\circ} 03^{\prime} \mathrm{N}, 40^{\circ} 16^{\prime} \mathrm{W}$, 5.V.1954, litter and soil sample, leg. W. Ivie, (3 sp.); USA-158, New York, Westchester County, Briancliff, 16.IV.1979, maple forest, leaf litter sample, leg. A. Young, (5 sp.); USA-306, Wash-

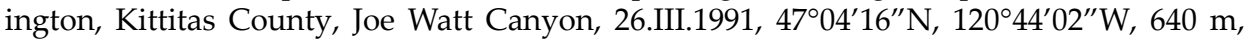
deciduous forest, Salix sp. litter sample, leg. R. Crawford, (1 sp.). Distribution: Nearctic species (NiedbaŁa 2002).

Oribotritia megale (Walker, 1965) - Material examined: USA-195, California, Humboldt County, Patrick's Point State Park, 21.VI.1991, 41 ${ }^{\circ} 08^{\prime} \mathrm{N}, 124^{\circ} 10^{\prime} \mathrm{W}$, coniferous forest, under Picea sitchensis, sample of litter and soil, leg. K. Horner, (1 sp.); USA-206, California, Del Norte County, Jedediah Smith State Park, 1.IV.1992, 41 ${ }^{\circ} 50^{\prime} \mathrm{N}, 124^{\circ} \mathrm{W}$, coniferous forest, under Tsuga heterophylla, sample of litter and soil, leg. K. Horner, (2 sp.); USA-207, California, Del Norte County, Jedediah Smith State Park, 1.IV.1992, 41 ${ }^{\circ} 50^{\prime} \mathrm{N}, 124^{\circ} \mathrm{W}$, coniferous forest, under Sequoia sempervirens, sample of litter and soil, leg. K. Horner, (3 sp.). Distribution: Nearctic species endemic to California (NiedbaŁa 2002). 
Oribotritia opipara Niedbała, 2002 - Material examined: USA-236, Tennessee, Blount County, Great Smoky Mountains National Park, Karst Quest, White Oak, Blowhole Cave Area, 26.VII.2006, sample of leaf litter outside of cave, leg. K. L. Felderhoff, (1 sp.); USA-238, Utah, Sevier County, Clingman's Dome, 24.VI.2006, deciduous bush, pitfall trap on right side of parking lot, leg. S. H. Allen, (1 sp.). Distribution: Nearctic species (NiedbaŁa 2002).

Maerkelotritia cryptopa (Banks, 1904) - Material examined: USA-212, Washington State, Little Kachess Lake, 5.X.1990, 47 $17^{\prime} 26^{\prime \prime} \mathrm{N}, 121^{\circ} 12^{\prime} 12^{\prime \prime} \mathrm{W}, 958$ m, coniferous forest, sample of litter and mosses, leg. M. Ranier, (1 sp.); USA-303, Washington, Kitsap County, Anderson Cove, 3.V.1992, 47 $7^{\circ} 3^{\prime} 58^{\prime \prime} \mathrm{N}, 122^{\circ} 58^{\prime} 05^{\prime \prime} \mathrm{W}, 9 \mathrm{~m}$, deciduous forest, Acer macrophyllum litter sample, leg. R. Crawford, (1 sp.), USA-308, Washington, Pierce County, Maplewood Spring, 20.IV.1992, 47 $10^{\prime} 30^{\prime \prime} \mathrm{N}, 122^{\circ} 18^{\prime} 58^{\prime \prime} \mathrm{W}, 15 \mathrm{~m}$, deciduous forest, Acer macrophyllum litter sample, leg. R. Crawford, (2 sp.). Distribution: Nearctic species restricted to west of the Nearctic Region (Niedbąa 2002).

Maerkelotritia kishidai (Aoki, 1958) - Material examined: USA-186, California, Del Norte County, Jedediah Smith State Park, 1.IV.1992, 41 50 'N, $124^{\circ} \mathrm{W}$, coniferous forest, under Sequoia sempervirens, sample of litter and soil, leg. K. Horner, (7 sp.); USA-192, Califor-

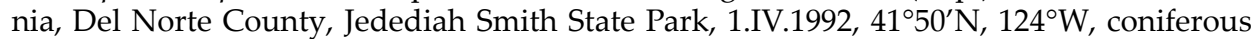
forest, under Tsuga heterophylla, sample of litter and soil, leg. K. Horner, (8 sp.). Distribution: Holarctic species (NiedbaŁa 2002, 2011).

Mesotritia flagelliformis (Ewing, 1909) - Material examined: USA-238, Utah, Sevier County, Clingman's Dome, 24.VI.2006, deciduous bush, pitfall trap on right side of parking lot, leg. S. H. Allen, (1 sp.). Distribution: Holarctic species (NiedbaŁa 2002).

Mesotritia nuda (Berlese, 1887) - Material examined: USA-154, Wisconsin, Milwaukee County, Kletzsch Peak, 6.VI.1978, mixed forest, sample of leaf litter, leg. J. P. Jass, (2 sp.). Distribution: Holarctic species (NiedbaŁa 2002, 2011).

Euphthiracarus alazon Walker, 1965 - Material examined: USA-192, California, Del Norte County, Jedediah Smith State Park, 1.IV.1992, 41 $500^{\prime} \mathrm{N}, 124^{\circ} \mathrm{W}$, coniferous forest, under Tsuga heterophylla, sample of litter and soil, leg. K. Horner, (3 sp.). Distribution: Nearctic species, endemic to California (NiedbaŁa 2002).

Euphthiracarus cernuus Walker, 1965 - Material examined: USA-301, Washington,

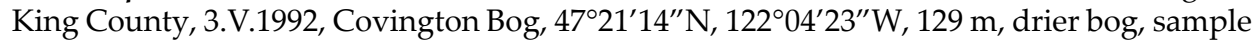
of Sphagnum sp., leg. R. Crawford, (3 sp.). Distribution: Nearctic species restricted to the west of the Nearctic Region (Niedbata 2002).

Euphthiracarus crassisetae Jacot, 1938 - Material examined: USA-192, California, Del Norte County, Jedediah Smith State Park, 1.IV.1992, $41^{\circ} 50^{\prime} \mathrm{N}, 124^{\circ} \mathrm{W}$, coniferous forest, under Tsuga heterophylla, sample of litter and soil, leg. K. Horner, (3 sp.). Distribution: Nearctic species, absent so far from central and southern parts of the Nearctic Region (Niedbata 2002).

Euphthiracarus cribrarius (Berlese, 1904) - Material examined: USA-445, North Carolina, Haywood County, Blue Ridge Parkway, across from Devils Courthouse, 17.VIII. $2005,32^{\circ} 42^{\prime} 40^{\prime \prime} \mathrm{N}, 39^{\circ} 05^{\prime} 00^{\prime \prime} \mathrm{W}, 1802 \mathrm{~m}$, mixed forest, sample of feathery moss and attached litter, leg. R. Bernard, (1 sp.). Distribution: Holarctic species (NiedbaєA 2002, 2011).

Euphthiracarus flavus (Ewing, 1908) - Material examined: USA-80, Pennsylvania, northeast of Jamison, Horseshoe Bend, Neshaming Creek, 5.V.1954, 7503’ N, 40 $16^{\prime} \mathrm{W}$, litter and soil sample, leg. W. Ivie, (3 sp.); USA-152, North Carolina, Macon County, Rich Mt., South of Highlands, 6.VI.1985, sample of leaf litter, leg. D. M. Hildebrandt, (1 sp.); USA-236, Tennessee, Blount County, Great Smoky Mountains National Park, Karst Quest, 
White Oak, Blowhole Cave Area, 26.VII.2006, sample of leaf litter outside of the cave, leg. K. L. Felderhoff, (1 sp.); USA-240, North Carolina, Graham County, Cherohala Skyway, 27.V.2007, $35^{\circ} 20^{\prime} 41^{\prime \prime} \mathrm{N}, 84^{\circ} 02^{\prime} 06^{\prime \prime} \mathrm{W}, 1403 \mathrm{~m}$, sample of soil under large hardwood lump, leg. A. Fjellberg, (4 sp.); USA-241, North Carolina, Graham County, Cherohala Skyway, 27.V.2007, 35 $20^{\prime} 12^{\prime \prime} \mathrm{N}, 84^{\circ} 02^{\prime} 06^{\prime \prime} \mathrm{W}, 1433 \mathrm{~m}$, harwood forest, sample of soil and litter, leg. A. Fjellberg, (9 sp.); USA-242, North Carolina, Monroe County, Cherohala, 27.V.2007, $35^{\circ} 20^{\prime} 59^{\prime \prime} \mathrm{N}, 84^{\circ} 04^{\prime} 03^{\prime \prime} \mathrm{W}$, mixed hardwood and hemlock forest, sample of dry litter, leg. A. Fjellberg, (25 sp.). Distribution: Nearctic species nearly broad, absent from the southwest of the Nearctic Region (Niedba€a 2002).

Euphthiracarus fulvus (Ewing, 1909) - Material examined: USA-318, Minnesota, Chippewa National Forest, Leech Lake, Ottertail, 26.IX.2010, $47^{\circ} 16^{\prime} 00^{\prime \prime} \mathrm{N}, 94^{\circ} 23^{\prime} 49^{\prime \prime} \mathrm{W}, 446$ $\mathrm{m}$, deciduous forest with dominant sugar maple, leaf litter and soil sample, leg. J. Schlaghamerský, (1 sp.). Distribution: Nearctic species, absent so far in southwest of the Nearctic Region (NiedbaŁa 2002).

Euphthiracarus longirostralis Walker, 1965 - Material examined: USA-192, California, Del Norte County, Jedediah Smith State Park, 1.IV.1992, 41 ${ }^{\circ} 50^{\prime} \mathrm{N}, 124^{\circ} \mathrm{W}$, coniferous forest, under Tsuga heterophylla, sample of litter and soil, leg. K. Horner, (8 sp.). Distribution: Nearctic species restricted to the west of the Nearctic Region (NiedbaŁa 2002).

Euphthiracarus parafusulus Niedbała, 2002 - Material examined: USA-445, North Carolina, Haywood County, Blue Ridge Parkway, across from Devils Courthouse, 17.VIII. $2005,32^{\circ} 42^{\prime} 40^{\prime \prime} \mathrm{N}, 39^{\circ} 05^{\prime} 00^{\prime \prime} \mathrm{W}, 1802 \mathrm{~m}$, mixed forest, sample of feathery moss and attached litter, leg. R. Bernard, (1 sp.). Distribution: Nearctic species with disjunctive pattern of distribution (NiedBaєA 2002).

Euphthiracarus polytretos Walker, 1965 - Material examined: USA-207, California, Del Norte County, Jedediah Smith State Park, 1.IV.1992, 41 ${ }^{\circ} 50^{\prime} \mathrm{N}, 124^{\circ} \mathrm{W}$, coniferous forest, under Sequoia sempervirens, sample of litter and soil, leg. K. Horner, (1 sp.). Distribution: Nearctic species, endemic to California (NiedbaŁa 2002).

Euphthiracarus punctulatus Jacot, 1930 - Material examined: USA-80, Pennsylvania, northeast of Jamison, Horseshoe Bend, Neshaming Creek, 5.V.1954, 7503' N, 40 $16^{\prime} \mathrm{W}$, litter and soil sample, leg. W. Ivie, (13 sp.). Distribution: Nearctic species restricted to eastern parts of the Nearcti Region (NiedbaŁa 2002).

Euphthiracarus tanythrix Walker, 1965 - Material examined: USA-235, Tennessee, Blount County, White Oak Sink, 28.VII.2006, deciduous forest, sample of leaf litter, leg. K. Felderhoff, (7 sp.). Distribution: Nearctic species (Niedba£A 2002).

Acrotritia ardua (C. L. Koch, 1841) - Material examined: USA-73, Pennsylvania,

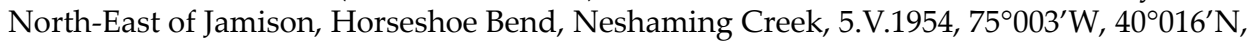
litter and soil sample, leg. W. Ivie, (30 sp.); USA-234, Tennessee, Blount County, Bull Cave Karst, 29.VII.2006, mixed forest, leaf litter sample, leg. P. J. Long, (2 sp.); USA-241, North

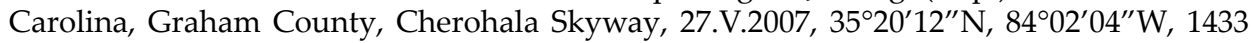
$\mathrm{m}$, hardwood forest, sample of soil and litter, leg. A. Fjellberg, (1 sp.); USA-326, Wisconsin, Tower Lake Wilderness, Chequamegon National Forest, 3.X.2010, 46 $26^{\prime} 03^{\prime \prime} \mathrm{N}, 91^{\circ} 19^{\prime} 36^{\prime \prime} \mathrm{W}$, $382 \mathrm{~m}$, deciduous forest with dominant sugar maple, leaf litter sample, leg. J. Schlaghamerský, (1 sp.); USA-330, Wisconsin, Tower Lake Wilderness, Chequamegon National Forest, 3.X.2010, $46^{\circ} 25^{\prime} 46^{\prime \prime} \mathrm{N}, 91^{\circ} 18^{\prime} 59^{\prime \prime} \mathrm{W}, 370 \mathrm{~m}$, deciduous forest with dominant sugar maple, sample of leaf litter, leg. J. Schlaghamerský, (1 sp.); USA-444, North Carolina, Haywood

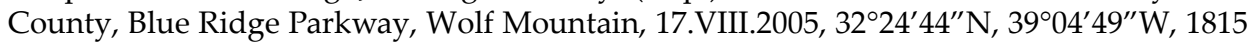
$\mathrm{m}$, sample of vegetation on side of seep, leg. R. Bernard, (31 sp.). Distribution: Semicosmopolitan species (NIEDBAEA 2011). 
Acrotritia curticephala (Jacot, 1938) - Material examined: USA-74, Pennsylvania, northeast of Jamison, Horseshoe Bend, Neshaming Creek, 5.V.1954, $75^{\circ} 03^{\prime} \mathrm{N}, 40^{\circ} 16^{\prime} \mathrm{W}$, litter and soil sample, leg. W. Ivie, (41 sp.). Distribution: Semicosmopolitan species not reported from the Afrotropical Region (NiedbaŁa 2004).

Acrotritia diaphoros (Niedbała, 2002) - Material examined: USA-157, Wisconsin, Milwaukee County, Jacobus Park, 8.IX.1978, mixed forest, leaf litter sample, leg. J. P. Jass, (1 sp.); USA-235, Tennessee, Blount County, White Oak Sink, 28.VII.2006, deciduous forest, sample of leaf litter, leg. K. Felderhoff, (5 sp.); USA-237, Pennsylvania, Cumberland County, Sequatchie River Valley, old route 28, 28.III.2006, base of rock wall, litter and soil sample, leg. J. K. Moulton, (1 sp.). Distribution: Nearctic species restricted to central and eastern parts of the Nearctic Region (NiedbaŁa 2002).

Acrotritia ornata (Niedbała, 2002) - Material examined: USA-240, North Carolina, Graham County, Cherohala Skyway, 27.V.2007, 3520'41”N, 8402'06”W, 1403 m, sample of soil under large hardwood lump, leg. A. Fjellberg, (1 sp.). Distribution: Nearctic species (Niedbata 2002).

Acrotritia scotti (Walker, 1965) - Material examined: USA-210, California, Del Norte County, Jedediah Smith State Park, 1.IV.1992, $41^{\circ} 50^{\prime} \mathrm{N}, 124^{\circ} \mathrm{W}$, coniferous forest, under Sequoia sempervirens, sample of litter and soil, leg. K. Horner, (12 sp.). Distribution: Nearctic species, endemic to California (Niedbaєa 2002).

Microtritia minima (Berlese, 1904) - Material examined: USA-242, North Carolina, Monroe County, Cherohala, 27.V.2007, 35 $20^{\prime} 59^{\prime \prime} \mathrm{N}, 84^{\circ} 04^{\prime} 03^{\prime \prime} \mathrm{W}$, mixed hardwood and hemlock forest, sample of dry litter, leg. A. Fjellberg, (55 sp.); USA-243, North Carolina, Graham County, Cherohala, 24.V.2007, $35^{\circ} 18^{\prime} 55^{\prime \prime} \mathrm{N}, 8^{\circ} 01^{\prime} 45^{\prime \prime} \mathrm{W}$, hardwood, sample of deep moist hardwood litter and soil, leg. A. Fjellberg, (7 sp.). Distribution: Holarctic species (NiedbaŁa 2002, 2011).

Microtritia simplex (Jacot, 1930) - Material examined: USA-192, California, Del Norte County, Jedediah Smith State Park, 1.IV.1992, $41^{\circ} 50^{\prime} \mathrm{N}, 124^{\circ} \mathrm{W}$, coniferous forest, under Tsuga heterophylla, sample of litter and soil, leg. K. Horner, (3 sp.); USA-210, California, Del Norte County, Jedediah Smith State Park, 1.IV.1992, $41^{\circ} 50^{\prime} \mathrm{N}, 124^{\circ} \mathrm{W}$, coniferous forest, under Sequoia sempervirens, sample of litter and soil, leg. K. Horner, (3 sp.); USA-330, Wisconsin, Tower Lake Wilderness, Chequamegon National Forest, 3.X.2010, $46^{\circ} 25^{\prime} 46^{\prime \prime} \mathrm{N}$, $91^{\circ} 18^{\prime} 59^{\prime \prime} \mathrm{W}, 370 \mathrm{~m}$, deciduous forest with dominant sugar maple, sample of leaf litter, leg. J. Schlaghamerský, (2 sp.). Distribution: Nearctic species (NiedbaŁa 2002).

Synichotritia caroli Walker, 1965 - Material examined: USA-192, California, Del Norte County, Jedediah Smith State Park, 1.IV.1992, 41 50 'N, $124^{\circ} \mathrm{W}$, coniferous forest, under Tsuga heterophylla, sample of litter and soil, leg. K. Horner, (59 sp.); USA-207, California, Del Norte County, Jedediah Smith State Park, 1.IV.1992, 415ㅇN N, $124^{\circ}$ W, coniferous forest, under Sequoia sempervirens, sample of litter and soil, leg. K. Horner, (1 sp.); USA-240, North

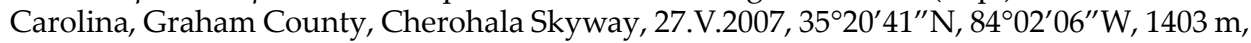
sample of soil under large hardwood lump, leg. A. Fjellberg, (11 sp.). Distribution: Nearctic species (NiedbaŁa 2002).

Synichotritia longipila Niedbała, 2002 - Material examined: USA-235, Tennessee, Blount County, White Oak Sink, 28.VII.2006, deciduous forest, sample of leaf litter, leg. K. Felderhoff, (2 sp.). Distribution: Nearctic species (NiedbaŁa 2002).

Synichotritia reticulata Niedbała et Starý, 2010 - Material examined: USA-242, North Carolina, Monroe County, Cherohala 27.V.2007, 35 $20^{\prime} 59^{\prime \prime} \mathrm{N}, 84^{\circ} 04^{\prime} 03^{\prime \prime} \mathrm{W}$, mixed hardwood and hemlock forest, sample of dry litter, leg. A. Fjellberg, (2 sp.). Distribution: Nearctic species perhaps endemic to North Carolina (NiedbaŁa \& StaRý 2010). 
Synichotritia spinulosa Walker, 1965 - Material examined: USA-186, California, Del Norte County, Jedediah Smith State Park, 1.IV.1992, 41 ${ }^{\circ} 50^{\prime} \mathrm{N}, 124^{\circ} \mathrm{W}$, coniferous forest, under Sequoia sempervirens, sample of litter and soil, leg. K. Horner, (116 sp.); USA-206, California, Del Norte County, Jedediah Smith State Park, 1.IV.1992, 41 ${ }^{\circ} 50^{\prime} \mathrm{N}, 124^{\circ} \mathrm{W}$, coniferous forest, under Tsuga heterophylla, sample of litter and soil, leg. K. Horner, (13 sp.). Distribution: Nearctic species (NiedbaєA 2002).

Phthiracarus aliquantus Niedbała, 1988 - Material examined: USA-186, California, Del Norte County, Jedediah Smith State Park, 1.IV.1992, $41^{\circ} 50^{\prime} \mathrm{N}, 124^{\circ} \mathrm{W}$, coniferous forest, under Sequoia sempervirens, sample of litter and soil, leg. K. Horner, (1 sp.); USA-235, Tennessee, Blount County, White Oak Sink, 28.VII.2006, deciduous forest, sample of leaf litter, leg. K. Felderhoff, (19 sp.); USA-237, Pennsylvania, Cumberland County, Sequatchie River Valley, old route 28, 28.III.2006, base of rock wall, leaf litter and soil sample, leg. J. K. Moulton, (1 sp.); USA-303, Washington, Kitsap County, Anderson Cove, 3.V.1992, $47^{\circ} 33^{\prime} 58^{\prime \prime} \mathrm{N}, 122^{\circ} 58^{\prime} 05^{\prime \prime} \mathrm{W}, 9 \mathrm{~m}$, deciduous forest, Acer macrophyllum litter sample, leg. R. Crawford, (3 sp.); USA-308, Washington, Pierce County, Maplewood Spring, 20.IV.1992, $47^{\circ} 10^{\prime} 30^{\prime \prime} \mathrm{N}, 122^{\circ} 18^{\prime} 58^{\prime \prime} \mathrm{W}, 15 \mathrm{~m}$, deciduous forest, Acer macrophyllum litter sample, leg. R. Crawford, (14 sp.). Distribution: Nearctic species (NiedbaŁa 2002).

Phthiracarus anonymus Grandjean, 1933 - Material examined: USA-195, California, Humboldt County, Patrick's Point State Park, 21.VI.1991, $41^{\circ} 08^{\prime} \mathrm{N}, 124^{\circ} 10^{\prime} \mathrm{W}$, coniferous forest, under Picea sitchensis, sample of litter and soil, leg. K. Horner, (1 sp.); USA-240, North Carolina, Graham County, Cherohala Skyway, 27.V.2007, 35 $20^{\prime} 41^{\prime \prime} \mathrm{N}, 84^{\circ} 02^{\prime} 06^{\prime \prime} \mathrm{W}$, $1403 \mathrm{~m}$, sample of soil under large hardwood lump, leg. A. Fjellberg, (2 sp.); USA-243, North Carolina, Graham County, Cherohala, 24.V.2007, 35 $18^{\prime} 55^{\prime \prime} \mathrm{N}, 84^{\circ} 01^{\prime} 45^{\prime \prime} \mathrm{W}$, hardwood, sample of deep moist hardwood litter and soil, leg. A. Fjellberg, (1 sp.). Distribution: Semicosmopolitan species (NiedbaŁa 2002).

Phthiracarus boresetosus Jacot, 1930 - Material examined: USA-212, Washington State, Little Kachess Lake, 5.X.1990, 47 $17^{\prime} 26^{\prime \prime} \mathrm{N}, 1^{\circ} 1^{\circ} 12^{\prime} 12^{\prime \prime} \mathrm{W}, 958$ m, coniferous forest, sample of litter and mosses, leg. M. Ranier, (2 sp.); USA-306, Washington, Kittitas County, Joe Watt Canyon, 26.III.1991, 47 $04^{\prime} 16^{\prime \prime} \mathrm{N}, 120^{\circ} 44^{\prime} 02^{\prime \prime} \mathrm{W}, 640 \mathrm{~m}$, deciduous forest, Salix sp. litter sample, leg. R. Crawford, (19 sp.); USA-326, Wisconsin, Tower Lake Wilderness, Chequamegon National Forest, 3.X.2010, $46^{\circ} 26^{\prime} 03^{\prime \prime} \mathrm{N}, 91^{\circ} 19^{\prime} 36^{\prime \prime} \mathrm{W}, 382 \mathrm{~m}$, deciduous forest with dominant sugar maple, leaf litter sample, leg. J. Schlaghamerský, (2 sp.); USA444, North Carolina, Haywood County, Blue Ridge Parkway, Wolf Mountain, 17.VIII.2005, $32^{\circ} 24^{\prime} 44^{\prime \prime} \mathrm{N}, 39^{\circ} 04^{\prime} 49^{\prime \prime} \mathrm{W}, 1815 \mathrm{~m}$, sample of vegetation on side of seep, leg. R. Bernard, (6 sp.). Distribution: Semicosmopolitan (NiedbaєA 2002, 2011).

Phthiracarus brevisetae Jacot, 1930 - Material examined: USA-206, California, Del Norte County, Jedediah Smith State Park, 1.IV.1992, 41 ${ }^{\circ} 50^{\prime} \mathrm{N}, 124^{\circ} \mathrm{W}$, coniferous forest, under Tsuga heterophylla, sample of litter and soil, leg. K. Horner, (1 sp.); USA-207, California, Del Norte County, Jedediah Smith State Park, 1.IV.1992, 41 ${ }^{\circ} 50^{\prime} \mathrm{N}, 124^{\circ} \mathrm{W}$, coniferous forest, under Sequoia sempervirens, sample of litter and soil, leg. K. Horner, (1 sp.); USA-240, North

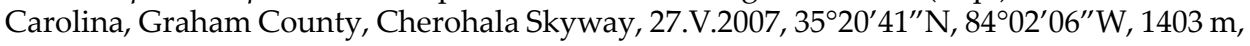
sample of soil under large hardwood lump, leg. A. Fjellberg, (2 sp.); USA-445, North Carolina, Haywood County, Blue Ridge Parkway, across from Devils Courthouse, 17.VIII.2005, $32^{\circ} 42^{\prime} 40^{\prime \prime} \mathrm{N}, 39^{\circ} 05^{\prime} 00^{\prime \prime} \mathrm{W}, 1802 \mathrm{~m}$, mixed forest, sample of feathery moss and attached litter, leg. R. Bernard, (2 sp.). Distribution: Nearctic species (NiedbaŁa 2002).

Phthiracarus bryobius Jacot, 1930 - Material examined: USA-238, Utah, Sevier County, Clingman's Dome, 24.VI.2006, deciduous bush, pitfall trap on right side of parking lot, leg. S. H. Allen, (4 sp.); USA-244, North Carolina, Haywood County, 24.V.2007, 35³5’06" N, 
$83^{\circ} 04^{\prime} 11^{\prime \prime} \mathrm{W}, 762 \mathrm{~m}$, maple forest, sample of material under bark of fallen maple stem, leg. A. Fjellberg, (5 sp.). Distribution: Holarctic species (NiedbaŁA 2002, 2010).

Phthiracarus cognatus Niedbała, 1988 - Material examined: USA-192, California, Del Norte County, Jedediah Smith State Park, 1.IV.1992, 41 ${ }^{\circ} 50^{\prime} \mathrm{N}, 124^{\circ} \mathrm{W}$, coniferous forest, under Tsuga heterophylla, sample of litter and soil, leg. K. Horner, (10 sp.); USA-235, Tennessee, Blount County, White Oak Sink, 28.VII.2006, deciduous forest, sample of leaf litter, leg. K. Felderhoff, (22 sp.); USA-236, Tennessee, Blount County, Great Smoky Mountains National Park, Karst Quest, White Oak, Blowhole Cave Area, 26.VII.2006, sample of leaf litter outside of cave, leg. K. L. Felderhoff, (3 sp.); USA-301, Washington, King County, Cov-

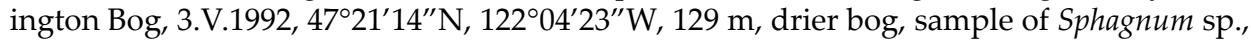
leg. R. Crawford, (1 sp.); USA-304, Washington, San Juan County, Spencer Spit State Park, 2.XI.1991, $48^{\circ} 32^{\prime} 06^{\prime \prime} \mathrm{N}, 122^{\circ} 51^{\prime} 25^{\prime \prime} \mathrm{W}, 18 \mathrm{~m}$, coniferous forest, sample of leaf litter, leg. R. Crawford, (8 sp.). Distribution: Nearctic species, restricted to northern and western parts of the Nearctic Region (NiedbaŁa 2002).

Phthiracarus compressus Jacot, 1930 - Material examined: USA-242, North Carolina, Monroe County, Cherohala 27.V.2007, 35 $20^{\prime} 59^{\prime \prime} \mathrm{N}, 84^{\circ} 04^{\prime} 03^{\prime \prime} \mathrm{W}$, mixed hardwood and hemlock forest, sample of dry litter, leg. A. Fjellberg, (9 sp.). Distribution: Holarctic species (NiedbaŁa 2002, 2010).

Phthiracarus globosus (C. L. Koch, 1841) - Material examined: USA-73, Pennsylvania, northeast of Jamison, Horseshoe Bend, Neshaming Creek, 5.V.1954, $75^{\circ} 03^{\prime} \mathrm{N}, 40^{\circ} 16^{\prime} \mathrm{W}$, litter and soil sample, leg. W. Ivie, (26 sp.); USA-154, Wisconsin, Milwaukee County, Kletzsch Peak, 6.VI.1978, mixed forest, sample of leaf litter, leg. J. P. Jass, (1 sp.); USA-156, Wisconsin, Milwaukee County, Kletzsch Peak, 10.VII.1978, mixed forest, leaf litter sample, leg. J. P. Jass, (1 sp.); USA-234, Tennessee, Blount County, Bull Cave Karst, 29.VII.2006, mixed forest, leaf litter sample, leg. P. J. Long, (19 sp.); USA-235, Tennessee, Blount County, White Oak Sink, 28.VII.2006, deciduous forest, sample of leaf litter, leg. K. Felderhoff, (37 sp.); USA-236, Tennessee, Blount County, Great Smoky Mountains National Park, Karst Quest, White Oak, Blowhole Cave Area, 26.VII.2006, sample of leaf litter outside of the cave, leg. K. Felderhoff, (1 sp.); USA-240, North Carolina, Graham County, Cherohala Skyway, 27.V.2007, $35^{\circ} 20^{\prime} 41^{\prime \prime} \mathrm{N}, 84^{\circ} 02^{\prime} 06^{\prime \prime} \mathrm{W}, 1403 \mathrm{~m}$, sample of soil under large hardwood lump, leg. A. Fjellberg, (1 sp.); USA-241, North Carolina, Graham County, Cherohala Skyway, 27.V.2007, 35 $20^{\prime} 12^{\prime \prime} \mathrm{N}, 84^{\circ} 02^{\prime} 04^{\prime \prime} \mathrm{W}, 1433 \mathrm{~m}$, hardwood forest, sample of soil and litter, leg. A. Fjellberg, (23 sp.); USA-314, Minnesota, Chippewa National Forest, Leech Lake, Ottertail, 26.IX.2010, $47^{\circ} 16^{\prime} 01^{\prime \prime} \mathrm{N}, 94^{\circ} 23^{\prime} 55^{\prime \prime} \mathrm{W}, 443 \mathrm{~m}$, deciduous forest with dominant sugar maple, leaf litter sample, leg. J. Schlaghamerský, (2 sp.); USA-445, North Carolina, Haywood County, Blue Ridge Parkway, across from Devils Courthouse, 17.VIII.2005, $32^{\circ} 42^{\prime} 40^{\prime \prime} \mathrm{N}$, $39^{\circ} 05^{\prime} 00^{\prime \prime} \mathrm{W}, 1802 \mathrm{~m}$, mixed forest, sample of feathery moss and attached litter, leg. R. Bernard, (15 sp.). Distribution: Holarctic species (NiedbaŁA 2002, 2010).

Phthiracarus irreprehensus Niedbała, 1988 - Material examined: USA-300, Washington, San Juan County, Orcas Island, Turtleback Mountain, 14.III.1992, 48 $38^{\prime} 38^{\prime \prime} \mathrm{N}$, $122^{\circ} 58^{\prime} 30^{\prime \prime} \mathrm{W}, 85 \mathrm{~m}$, oak forest, Quercus garryana leaf litter sample, leg. R. Crawford, (1 sp.). Distribution: Nearctic species restricted to the west of the Nearctic Region (NiEdbaŁa 2002).

Phthiracarus japonicus Aoki, 1958 - Material examined: USA-207, California, Del Norte County, Jedediah Smith State Park, 1.IV.1992, 41 ${ }^{\circ} 50^{\prime} \mathrm{N}, 124^{\circ} \mathrm{W}$, coniferous forest, under Sequoia sempervirens, sample of litter and soil, leg. K. Horner, (1 sp.), USA-300, Washington, San Juan County, Orcas Island, Turtleback Mountain, 14.III.1992, 48 $38^{\prime} 38^{\prime \prime} \mathrm{N}$, $122^{\circ} 58^{\prime} 30^{\prime \prime} \mathrm{W}, 85 \mathrm{~m}$, oak forest, Quercus garryana litter sample, leg. R. Crawford, (17 sp.), USA-304, Washington, San Juan County, Spencer Spit State Park, 2. XI. 1991, 48 $32^{\prime} 06^{\prime \prime}$ N, 
$122^{\circ} 51^{\prime} 25^{\prime \prime} \mathrm{W}, 18 \mathrm{~m}$, coniferous forest, sample of coniferous litter, leg. R. Crawford, (14 sp.), USA-308, Washington, Pierce County, Maplewood Spring, 20.IV.1992, 47¹0'30”N, $122^{\circ} 18^{\prime} 58^{\prime \prime} \mathrm{W}, 15 \mathrm{~m}$, deciduous forest, Acer macrophyllum litter sample, leg. R. Crawford, (20 sp.). Distribution: Holarctic species (NiedbaŁa 2002, 2010).

Phthiracarus longulus (C. L. Koch, 1841) - Material examined: USA-80, Pennsylvania, northeast of Jamison, Horseshoe Bend, Neshaming Creek, 5.V.1954, 7503' N, 40 $16^{\prime} \mathrm{W}$, litter and soil sample, leg. W. Ivie, (5 sp.); USA-195, California, Humboldt County, Patrick's Point State Park, 21.VI.1991, $41^{\circ} 08^{\prime} \mathrm{N}, 124^{\circ} 10^{\prime} \mathrm{W}$, coniferous forest, under Picea sitchensis, sample of litter and soil, leg. K. Horner, (17 sp.); USA-210, California, Del Norte County, Jedediah Smith State Park, 1.IV.1992, $41^{\circ} 50^{\prime} \mathrm{N}, 124^{\circ} \mathrm{W}$, coniferous forest, under Sequoia sempervirens, sample of litter and soil, leg. K. Horner, (1 sp.); USA-236, Tennessee, Blount County, Great Smoky Mountains National Park, Karst Quest, White Oak, Blowhole Cave Area, 26.VII.2006, sample of leaf litter outside of the cave, leg. K. Felderhoff, (1 sp.); USA-238, Utah, Sevier County, Clingman's Dome, 24.VI.2006, deciduous bush, pitfall trap on right side of parking lot, leg. S. H. Allen, (1 sp.). Distribution: Holarctic species (NiedbaŁa 2002).

Phthiracarus modestus Niedbała, 1988 - Material examined: USA-192, California, Del Norte County, Jedediah Smith State Park, 1.IV.1992, 41 ${ }^{\circ} 50^{\prime} \mathrm{N}, 124^{\circ} \mathrm{W}$, coniferous forest, under Tsuga heterophylla, sample of litter and soil, leg. K. Horner, (33 sp.); USA-207, California, Del Norte County, Jedediah Smith State Park, 1.IV.1992, 41 ${ }^{\circ} 50^{\prime} \mathrm{N}, 1^{\circ} 4^{\circ} \mathrm{W}$, coniferous forest, under Sequoia sempervirens, sample of litter and soil, leg. K. Horner, (4 sp.). Distribution: Nearctic species restricted so far to the West of the Nearctic Region (Niedbata 2002).

Phthiracarus pusillus Niedbała, 2001 - Material examined: USA-234, Tennessee, Blount County, Bull Cave Karst, 29.VII.2006, mixed forest, leaf litter sample, leg. P. J. Long, (3 sp.). Distribution: Nearctic species (NiedbaŁa 2002).

Phthiracarus sarahae Jacot, 1930 - Material examined: USA-300, Washington, San

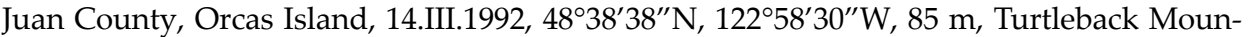
tain, oak forest, Quercus garryana litter sample, leg. R. Crawford, (1 sp.); USA-445, North Carolina, Haywood County, Blue Ridge Parkway, across from Devils Courthouse, 17.VIII.2005, $32^{\circ} 42^{\prime} 40^{\prime \prime} \mathrm{N}, 39^{\circ} 05^{\prime} 00^{\prime \prime} \mathrm{W}, 1802 \mathrm{~m}$, mixed forest, sample of feathery moss and attached litter, leg. R. Bernard, (2 sp.). Distribution: Nearctic species restricted to eastern part of the Nearctic Region (NiedbaŁa 2002).

Phthiracarus setosus (Banks, 1895) - Material examined: USA-154, USA, Wisconsin, Milwaukee County, Kletzsch Peak, 6.VI.1978, mixed forest, sample of leaf litter, leg. J. P. Jass, (1 sp.); USA-156, Wisconsin, Milwaukee County, Kletzsch Peak, 10.VII.1978, mixed forest, leaf litter sample, leg. J. P. Jass, (1 sp.); USA-234, Tennessee, Blount County, Bull Cave Karst, 29.VII.2006, mixed forest, leaf litter sample, leg. P. J. Long, (5 sp.). Distribution: Holarctic species (Niedba€A 2002, 2010).

Steganacarus (Rhacaplacarus) thoreaui (Jacot, 1930) - Material examined: USA-445, North Carolina, Haywood County, Blue Ridge Parkway, across from Devils Courthouse, 17.VIII.2005, 32 $42^{\prime} 40^{\prime \prime} \mathrm{N}, 39^{\circ} 05^{\prime} 00^{\prime \prime} \mathrm{W}, 1802 \mathrm{~m}$, mixed forest, sample of feathery moss and attached litter, leg. R. Bernard, (20 sp.). Distribution: Nearctic species absent so far from west of the Nearctic Region. (Niedba€a 2002).

Austrophthiracarus olivaceus (Jacot, 1929) - Material examined: USA-240, North

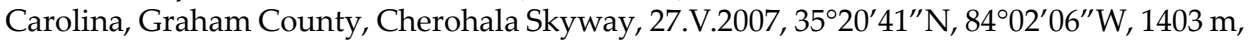
sample of soil under large hardwood lump, leg. A. Fjellberg, (1 sp.). Distribution: Nearctic species absent in south-east of the Nearctic Region (Niedbaєa 2002). 
Atropacarus (Atropacarus) absimilis (Niedbała, 1982) - Material examined: USA235, Tennessee, Blount County, White Oak Sink, 28.VII.2006, deciduous forest, sample of leaf litter, leg. K. Felderhoff, (41 sp.); USA-237, Pennsylvania, Cumberland County, Sequatchie River Valley, old route 28, 28.III.2006, base of rock wall, litter and soil sample, leg. J. K. Moulton, (1 sp.); USA-244, North Carolina, Haywood County, 24.V.2007, 35³5’06”'N, $83^{\circ} 04^{\prime} 11^{\prime \prime} \mathrm{W}, 762 \mathrm{~m}$, maple forest, sample of material under bark of fallen maple stem, leg. A. Fjellberg, (1 sp.). Distribution: Nearctic species restricted to southeast of the Nearctic Region (NiedbaŁa 2002).

Atropacarus (Atropacarus) striculus (C. L. Koch, 1835) - Material examined: USA157, Wisconsin, Milwaukee County, Jacobus Park, 8.IX.1978, mixed forest, leaf litter sample, leg. J. P. Jass, (1 sp.); USA-234, Tennessee, Blount County, Bull Cave Karst, 29.VII.2006, mixed forest, leaf litter sample, leg. P. J. Long, (22 sp.); USA-235, Tennessee, Blount County, White Oak Sink, 28.VII.2006, deciduous forest, sample of leaf litter, leg. K. Felderhoff, (1 sp.); USA-240, North Carolina, Graham County, Cherohala Skyway, 27.V.2007, 35 $20^{\prime} 41^{\prime \prime} \mathrm{N}$, $84^{\circ} 02^{\prime} 06^{\prime \prime} \mathrm{W}, 1403 \mathrm{~m}$, sample of soil under large hardwood lump, leg. A. Fjellberg, (7 sp.); USA-241, North Carolina, Graham County, Cherohala Skyway, 27.V.2007, 35²0'12"N, $84^{\circ} 02^{\prime} 04^{\prime \prime} \mathrm{W}, 1433 \mathrm{~m}$, hardwood forest, sample of soil and litter, leg. A. Fjellberg, (305 sp.); USA-242, North Carolina, Monroe County, Cherohala 27.V.2007, 35 $20^{\prime} 59^{\prime \prime} \mathrm{N}, 84^{\circ} 04^{\prime} 03^{\prime \prime} \mathrm{W}$, mixed hardwood and hemlock forest, sample of dry litter, leg. A. Fjellberg, (4 sp.); USA243, North Carolina, Graham County, Cherohala, 24.V.2007, 35 $18^{\circ} 55^{\prime \prime} \mathrm{N}, 84^{\circ} 01^{\prime} 45^{\prime \prime} \mathrm{W}$, hardwood, sample of deep moist hardwood litter and soil, leg. A. Fjellberg, (18 sp.); USA-

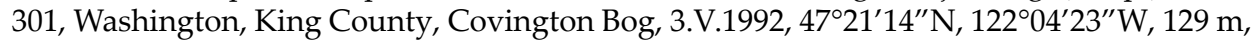
drier bog, sample of Sphagnum sp., leg. R. Crawford, (1 sp.); USA-306, Washington, Kittitas County, Joe Watt Canyon, 26.III.1991, 47 $04^{\prime} 16^{\prime \prime} \mathrm{N}, 120^{\circ} 44^{\prime} 02^{\prime \prime} \mathrm{W}, 640 \mathrm{~m}$, deciduous forest, Salix sp. litter sample, leg. R. Crawford, (31 sp.); USA-309, Washington, Mason County, Belfair Bog, 20.III.1992, 116 m, sample of Sphagnum sp. from wet bog, leg. R. Crawford, (6 sp.); USA-310, Minnesota, Chippewa National Forest, Leech Lake, Ottertail, 26.IX.2010, $47^{\circ} 15^{\prime} 59^{\prime \prime} \mathrm{N}, 94^{\circ} 23^{\prime} 37^{\prime \prime} \mathrm{W}, 449 \mathrm{~m}$, deciduous forest with dominant sugar maple, leaf litter sample, leg. J. Schlaghamerský, (13 sp.); USA-445, North Carolina, Haywood County, Blue Ridge Parkway, across from Devils Courthouse, 17.VIII.2005, 32 $42^{\prime} 40^{\prime \prime} \mathrm{N}, 39^{\circ} 05^{\prime} 00^{\prime \prime} \mathrm{W}$, $1802 \mathrm{~m}$, mixed forest, sample of feathery moss and attached litter, leg. R. Bernard, (52 sp.). Distribution: Holarctic species (NiedbaŁA 2002, 2011).

Atropacarus (Atropacarus) terrapene (Jacot, 1937) - Material examined: USA-234, Tennessee, Blount County, Bull Cave Karst, 29.VII.2006, mixed forest, leaf litter sample, leg. P. J. Long, (3 sp.). Distribution: Nearctic species restricted to central and eastern parts of the Nearctic Region (Niedba€a 2002).

\section{Records from Cuba}

Mesotritia elegantula Starý, 1992 - Material examined: K-322, Cuba, Province Habana, Arroyo Bermejo, 25.X.1981, semideciduous forest, North slope, leaf litter sample, leg. J. Rusek, (1 sp.). Distribution: Neotropical species, endemic to Cuba (Niedba£a 2004).

Acrotritia dikra Niedbała et Schatz, 1996 - Material examined: K-271, Cuba, Province Cienfuegos, Yaguramas, 2.X.1981, deciduous forest, decaying wood sample, leg. J. Rusek, (2 sp.). Distribution: Neotropical species probably introduced to Illinois, USA (NiedbaŁA 2004). 
Records from Jamaica

Phthiracarus pandus Niedbała, 2004 - Material examined: JAM-44, Jamaica, Blue Mountains, 15.IV.1992, montane deciduous forest, leaf litter sample, leg. R. Hogervorst, (1 sp.). Distribution: Neotropical species endemic to Jamaica (NiEdbAŁA 2004).

\section{Records from Brazil}

Acrotritia dikra Niedbała et Schatz, 1996 - Material examined: Brazil, Rio de Janeiro

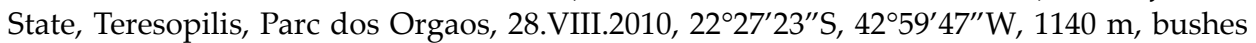
and perennial plants, leaf litter sample, leg. W. Witaliński, (1 sp.); Brazil, Igarassu near Recife, 25.VIII.2010, 57 $48^{\prime} 56^{\prime \prime}$ S, 34 $57^{\prime} 16^{\prime \prime} \mathrm{W}, 1 \mathrm{~m}$, restoration site for sloughts, litter along the road in Atlantic rain forest, leg. W. Witaliński, (2 sp.). Distribution: Neotropical species probably introduced to Illinois, USA (NiedbaŁa 2004).

Phthiracarus anonymus Grandjean, 1933 - Material examined: Brazil, Rio de Janeiro

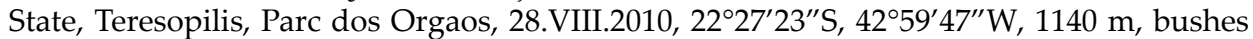
and perennial plants, leaf litter sample, leg. W. Witaliński, (1 sp.). Distribution: Semicosmopolitan species (NiedbaŁa 2011).

Phthiracarus phoxos Niedbała, 2004 - Material examined: BR-046, Brazil, São Paulo State, São Paulo, Instituto Butantan, $50 \mathrm{~m}$ from the Museum of Microbiology, 7.XI.2002, secondary Atlantic forest, sample of wet leaf litter, leg. J. Starý, (2 sp.). Distribution: Neotropical species (NiedbaŁa 2004).

Steganacarus (Rhacaplacarus) brasiliensis (Niedbała, 1988) - Material examined: BR-046, Brazil, São Paulo State, São Paulo, Instituto Butantan, $50 \mathrm{~m}$ from the Museum of Microbiology, 7.XI.2002, secondary Atlantic forest, sample of wet leaf litter, leg. J. Starý, (3 sp.). Distribution: Neotropical species, restricted to Central America and Brazil (NiedbatA 2004).

Steganacarus (Rhacaplacarus) sedecimus Niedbała, 2004 - Material examined: Bra-

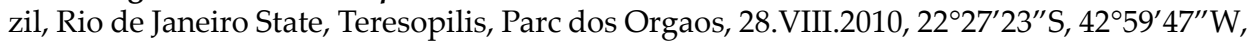
$1140 \mathrm{~m}$, bushes and perennial plants, leaf litter sample, leg. W. Witaliński, (4 sp.). Distribution: Neotropical species restricted to Brazil and Peru (Niedba£a 2004).

Steganacarus (Steganacarus) rafalskii (Niedbała, 1981) - Material examined: Brazil, Rio de Janeiro State, Teresopilis, Parc dos Orgaos, 28.VIII.2010, 22 $27^{\prime} 23^{\prime \prime} \mathrm{S}, 42^{\circ} 59^{\prime} 47^{\prime \prime} \mathrm{W}$, $1140 \mathrm{~m}$, bushes and perennial plants, leaf litter sample, leg. W. Witaliński, (1 sp.). Distribution: Neotropical species, perhaps endemic to southeast of Brazil (Niedba£a 2004).

Austrophthiracarus elizabethae (Niedbała, 1988) - Material examined: Brazil, Igarassu near Recife, 25.VIII.2010, 57 $48^{\prime} 56^{\prime \prime}$ S, 34 $4^{\circ} 7^{\prime} 16^{\prime \prime} \mathrm{W}, 1 \mathrm{~m}$, restoration site for sloughts, litter along the road in Atlantic rain forest, leg. W. Witaliński, (6 sp.). Distribution: Neotropical species, perhaps endemic to Brazil (NiedbaŁa 2004).

Atropacarus (Hoplophorella) hamatus (Ewing, 1909) - Material examined: Brazil, Igarassu near Recife, 25.VIII.2010, 57 $48^{\prime} 56^{\prime \prime}$ S, 34 $4^{\circ} 7^{\prime} 16^{\prime \prime} \mathrm{W}, 1 \mathrm{~m}$, restoration site for sloughts, litter along the road in Atlantic rain forest, leg. W. Witaliński, (2 sp.); BR-046, Brazil, São Paulo State, São Paulo, Instituto Butantan, $50 \mathrm{~m}$ from the Museum of Microbiology, 7.XI.2002, secondary Atlantic forest, sample of wet leaf litter, leg. J. Starý, (1 sp.). Distribution: Neotropical species (NiedbaŁa 2004). 
Records from Galapagos Islands

Oribotritia didyma Niedbała et Schatz, 1996 - Material examined: GLG-08, Galapagos Islands, Floreana Island, Asilo del la Paz, 17.V.1975, spring in the forest, sample of decaying wood and fern litter and rhizosphere, leg. H. Franz, (3 sp.). Distribution: Mesoamerica and Galapagos Islands (NiedbaŁa 2004).

Indotritia bellingeri Niedbała et Schatz, 1996 - Material examined: GLG-11, Galapagos Islands, Isabela Island, Bosque de Jaboncillo near Recinto San Antonio, under Santo Tomas, 29.V.1974, forest litter sample, leg. H. Franz, (2 sp.). Distribution: Pan-neotropical species (NiedbaєA 2004).

Indotritia retusa Niedbała et Schatz, 1996 - Material examined: GLG-03, Galapagos Islands, San Cristobal Island, Cerro San Joaquin, 4.V.1975, bush, sample of leaf litter, leg. H. Franz, (1 sp.); GLG-04, Galapagos Islands, San Cristobal Island, 5.V.1975, ravine with arborescent fern, sample of fern litter, leg. H. Franz, (94 sp.); GLG-08, Galapagos Islands, Floreana Island, Asilo del la Paz, 17.V.1975, spring in the forest, sample of decaying wood and fern litter and rhizosphere, leg. H. Franz, (39 sp.); GLG-11, Galapagos Islands, Isabela Island, Bosque de Jaboncillo near Recinto San Antonio, under Santo Tomas, 29.V.1974, forest litter sample, leg. H. Franz, (1 sp.); GLG-12, Galapagos Islands, Isabela Island, Volcan Cerro Negro, 30.V.1975, growth in lava depression in volcano margin, sample of moss and fern rhizosphere, leg. H. Franz, (14 sp.). Distribution: Neotropical species, Galapagos Islands and the Antilles only (NiedbaŁa 2004).

Acrotritia vestita (Berlese, 1913)- Material examined: GLG-04, Galapagos Islands, San Cristobal Island, 5.V.1975, ravine with arborescent fern, sample of fern litter, leg. H. Franz, (1 sp.). Distribution: Pantropical species (NiedbaŁa 2011).

\section{HOMONYM TAXON}

Austrophthiracarus longisetosus Niedbała et Starý, 2015 from Bolivia is a junior homonym of Austrophthiracarus longisetosus Lieu et Chen, 2014 from China. Therefore we replace it with Austrophthiracarus longisetus nom. n.

Acknowledgements - We would like to thank the anonymous referees for constructive criticism of the manuscript and many collectors of soil samples in many countries of the world. The study was partly supported by the Academy of Sciences of the Czech Republic, Research Plan No. AV0Z60660521.

\section{REFERENCES}

Liv, D. \& Chen, J. (2014) Descriptions of two new species of Austrophthiracarus Balogh et Mahunka, a newly recorded genus of ptyctimous mites from China (Acari: Oribatida: Phthiracaridae). Annales Zoologici 64(2): 267-272.

Mahunka, S. (1996) Oribatids from Sarawak I. (Acari: Oribatida). New and interesting mites from the Geneva Museum, LXXVIII. Revue suisse de Zoologie 103(1): 259-282. 
Manunka, S. (2008) More oribatids from Thailand (Acari: Oribatida). Revue suisse de Zoologie 115(4): 623-649.

Myers, N., Mittermeyer, R. A., Mittermeyer, C. G., da Fonseca, G. A. B. \& Kent, K. (2000) Biodiversity hotspots for conservation priorities. Nature 403: 853-858.

NiedbatA, W. (1982) Nouveaux Phthiracaridae tropicaux (Acari, Oribatida). Bulletin Entomologique de Pologne 52: 189-229.

NiedbaŁA, W. (1988a) Deux nouveaux Phthiracaridae des Comores (Acari, Oribatida). Journal of African Zoology 102: 79-83.

NiedbatA, W. (1988b) Nouveaux Phthiracaroidea (Acari, Oribatida) Neotropicales. Redia 71(1): 115-138.

NiedbaŁA, W. (2000) The ptyctimous mites fauna of the Oriental and Australian Regions and their centres of origin (Acari: Oribatida). Genus, Supplement: 1-493.

NiedbaŁA, W. (2001) Study on the diversity of ptyctimous mites (Acari: Oribatida) and quest for centres of its origin: the fauna of the Ethiopian Region. Monographs of the Upper Silesian Museum 3: 1-245.

Niedba€A, W. (2002) Ptyctimous mites (Acari, Oribatida) of the Nearctic Region. Monographs of the Upper Silesian Museum 4: 1-261.

NiedbatA, W. (2003) Ptyctimous mites (Acari: Oribatida) of Costa Rica. Annales Zoologici 53(2): 259-334.

NiedbaŁa, W. (2004) Ptyctimous mites (Acari, Oribatida) of the Neotropical Region. Annales Zoologici 54(1): 1-288.

NiedbaŁA, W. (2006) Four new species of Ptyctimous mites (Acari: Oribatida) from Mesoamerica. Annales Zoologici 56(4): 791-797.

Niedba€A, W. (2007) A new species of ptyctimous mites (Acari: Oribatida) from the Nearctic Region. Canadian Entomologist 139: 510-512. doi: 10.4039/n06-866

NiedbąA, W. (2010) Oribatida inferiors - Macropylina. Ptyctimous mites. Pp. 348-353. In: Gerlach, J. \& Marusik, Y. (eds): Arachnida and Myriapoda of the Seychelles Islands. Siri Scietific Press, Manchester.

NiedbaŁa, W. (2011) Ptyctimous mites (Acari, Oribatida) of the Palaearctic Region. Systematic part. Fauna Mundi 4: 1-472.

Niedbata, W. \& Corpuz-Raros, L. A. (1998) Ptyctimous mites (Acari, Oribatida) from the Philippines. Philippine Agriculturist 81(1-2): 1-58.

NiedbaeA, W., Corpuz-Raros, L. A. \& Gruezo, W. S. M., (2006) Ptyctimous mites mainly from Samar Island of the Philippines (Acari: Oribatida). Genus 17(3): 449-470.

NiedbaŁA, W. \& Ermilov, S. G. (2012) Ptyctimous mites (Acari, Oribatida) from southern Ethiopia with description of three new species. Systematic \& Applied Acarology 17(2): 182-190. doi: 10.11158/saa.17.2.4

Niedbąa, W. \& Starý, J. (2010) Three Holarctic new species of ptyctimous mites (Acari, Oribatida). Zootaxa 2625: 63-68.

NiedbaŁa, W. \& Starý, J. (2015) Three new species of the family Phthiracaridae (Acari, Oribatida) from Bolivia. Zootaxa 3918: 113-127. doi: 10.11646/zootaxa.3918.1.6

OjedA, M. (1985) Nueva especie de Protophthiracarus (Oribatei: Phthiracaridae) de Venezuela. Folia Entomológica Mexicana 64: 93-100.

Walker, N. A. (1965) Euphthiracaroidea of California Sequoia litter with a reclassification of the families and genera of the World (Acarina: Oribatei). Fort Hays Studies 3: 1-154.

Revised version received December 10, 2014, accepted March 3, 2015, published May 29, 2015 